\title{
Material Point Method for crack propagation in anisotropic media - A phase field approach
}

\author{
E. G. Kakouris · S.P. Triantafyllou
}

Received: date / Accepted: date

\begin{abstract}
A novel phase field formulation implemented within a material point method setting is developed to address brittle fracture simulation in anisotropic media. The case of strong anisotropy in the crack surface energy is treated by considering an appropriate variational, i.e., phase field approach. Material point method is utilized to efficiently treat the resulting coupled governing equations. The brittle fracture governing equations are defined at a set of Lagrangian material points and subsequently interpolated at the nodes of a fixed Eulerian mesh where solution is performed. As a result, the quality of the solution does not depend on the quality of the underlying finite element mesh and is relieved from mesh-distortion errors. The efficiency and validity of the proposed method is assessed through a set of benchmark problems.
\end{abstract}

Keywords brittle fracture $\cdot$ anisotropy $\cdot$ phase field $\cdot$ material point method

\section{Introduction}

Anisotropy occurs naturally in several material such as timber, geo-materials, and biological tissues due to inherent heterogeneities, inclusions and/ or constituent distribution that in many cases are distributed in a random fashion. Industrial materials may also exhibit anisotropy due to their controlled or partially controlled manufacturing process as in the case of laminated composites. Anisotropy significantly affects crack formation. In cases of even weak anisotropy, stress concentrations pertinent to crack initiation and propagation will preferentially develop along the weak directions of the deformable medium. This intriguing mechanical response is being often highlighted in experiments.

S. P. Triantafyllou · E.G. Kakouris

Centre for Structural Engineering and Informatics

The University of Nottingham

NG7 2RD, UK

Tel.: +44 (0) 1159514108

E-mail: savvas.triantafyllou@nottingham.ac.uk 
Very recently variational methods have been introduced, in particular phasefield approximations, providing a robust mathematical framework for the description of discontinuities [14]. Phase-field fracture models represent cracks by means of an additional continuous field (termed the phase-field) that is defined over the entire deformable domain and smoothly varies from zero (inside the crack) to one (away from the crack). Relieved from the singularity bottle-neck, phase-field models overcome the need for algorithmic tracking of discontinuities. Consequently, phase-field method is able to handle complex crack paths, including crack branching and crack merging that are commonly observed in dynamic brittle fracture problems [10]. Phase-field description of brittle and ductile fracture has been extensively used in the literature for the isotropic case (see, e.g., [1,23]). Very recently, extensions have been introduced to tackle fracture albeit in particular cases, i.e., crystals demonstrating cubic symmetry $[12,21]$, arterial walls under the assumption of exponential anisotropy [16], and cleavage fracture pertaining to crystal structures [34]. In this work, a mathematical framework is formulated to address the case of phase-field description of brittle fracture for the general case of a fully anisotropic material.

Crack initiation and propagation has been treated within the realm of particle based methods such as discrete element methods [32], smooth particle hydrodynamics [7] and peridynamics [8]. Although such approaches are able to resolve crack paths with extreme accuracy both in the quasi-static and dynamic regime, they are computationally taxing as a large number of particles is required to highlight the underlying interactions. Furthermore, a set of additional procedures is required to achieve convergence, i.e., high-order integration schemes and neighbour searching [28].

Material Point Method (MPM) [37] has emerged as an extension of ParticleIn-Cell methods, combining concepts pertinent to both the Eulerian and Lagrangian description of classical mechanics [6]. To this point, MPM has been proven advantageous in the analysis of large scale problems involving material and geometric non-linearities, especially within the context of coupled field problems, e.g., poro-mechanics [19,3]. In MPM, the deformable domain is discretised into a set of material points that are moving within a fixed (Eulerian) computational grid. Solution of the governing equations is performed in the Eulerian grid utilizing appropriate interpolation functions.

MPM has been found to offer significant computational advantages when compared to purely meshless methods since it does not require time-consuming neighbour searching. MPM has been proven to be sensitive to the so-called cell crossing error manifested as an oscillation in the stress field when a material point moves from one back-ground cell to another [36,35]. This has been treated in the literature by various methods, most notably by introducing the Generalized Interpolation Material Point Method (GIMPM) [4] or more recently with a convected particle domain interpolation technique (CPDI) [30, 29]. In this work, the Eulerian Grid is formulated by high-order B-Splines shape functions to treat this issue due to their implementational efficiency [36, 35]. Numerical investigations in this work show that the use of higher-order 
B-splines further improves the convergence rates of the coupled phase-field formulation for crack propagation introduced.

Material Point Methods are advantageous when used to resolve crack paths for two reasons. First, sharp discontinuities in the displacement field can be naturally treated, as response is monitored at material points that move within a fixed Eulerian grid. Thus, no re-meshing and or enrichment of the underlying finite element mesh is required as the latter is relieved from the detrimental effects of mesh distortion. In [33] and also [39] decohesion was treated by introducing a cohesive material constitutive framework at the material point level. Brittle fracture within an MPM setting was examined for the first time in [26] although considering only the case of pre-existing, i.e., explicit, crack geometries by allowing multiple velocity fields to be defined at the background grid. More recently, cohesive modelling approaches have been introduced in an effort to further generalize the applicability of the MPM for problems pertinent to arbitrary crack paths $[13,5,41,31]$. A continuum damage mechanics MPM methodology has also been introduced to tackle damage initiation and propagation in the anisotropic regime [27]. Phase field simulation of brittle fracture of isotropic materials has been introduced by the authors within an MPM setting in [20]. In this work, MPM is reformulated to incorporate a general anisotropic phase-field description for crack propagation and its merits and bottlenecks are assessed as compared to a standard Finite Element (FEM) implementation.

This work is organized as follows: the governing equations of brittle fracture for the general case of an anisotropic medium together with the anisotropic phase-field model developed in this work are introduced in Section 2. Next, the Material Point Method implementation is discussed in Section 3. Finally, in Section 4 a set of benchmark tests is presented and the efficiency of the proposed formulation is assessed. The proposed MPM framework for fracture in anisotropic media is verified against an anisotropic finite element phase-field implementation.

\section{Phase field modelling for anisotropic fracture}

\subsection{Preliminaries}

In the following, the case of an arbitrary deformable domain $\Omega$ is considered. The domain is sufficiently kinematically restrained along the boundary $\partial \Omega_{\bar{u}} \subset$ $\partial \Omega$. Furthermore, it is assumed that a crack path $\Gamma$ evolves, under the action of a set of tractions $\overline{\boldsymbol{t}}$ applied on $\partial \Omega_{\bar{t}} \in \partial \Omega$ and body forces $\boldsymbol{b}$. In his theory of brittle fracture, Griffith [15] postulated that the potential energy of the deformable domain $\Omega$ with an evolving crack path is defined as

$$
\Psi_{p o t}=\Psi_{e l}+\Psi_{f}=\int_{\Omega} \psi_{e l}(\varepsilon) d \Omega+\int_{\Gamma} \mathcal{G}_{c}(\theta) d \Gamma
$$




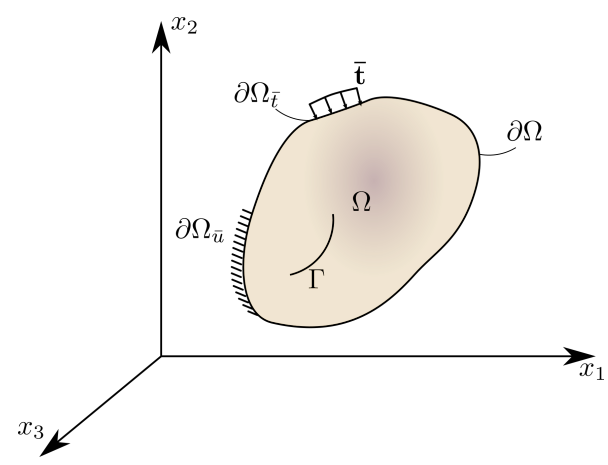

Fig. 1 Deformable domain $\Omega$

where $\Psi_{e l}$ and $\Psi_{f}$ are the elastic strain energy and the fracture energy (termed herein as surface energy for brevity) respectively. Furthermore, $\psi_{e l}(\varepsilon)$ corresponds to the elastic energy density, whereas $\varepsilon$ is the symmetric strain tensor which under the small strain assumption is defined as

$$
\varepsilon=\frac{1}{2}\left(\nabla \mathbf{u}+\nabla \mathbf{u}^{T}\right)
$$

where the $(\nabla)$ stands for the gradient operator and $\mathbf{u}(\mathbf{x}, t)$ for the displacement field.

The term $\mathcal{G}_{c}(\theta)$ corresponds to the critical fracture energy density released (surface energy density) over the crack path $\Gamma$. In the anisotropic case, this is considered to explicitly depend on the angle of orientation of the crack path $\theta$. Thus, the integral expression representing the fracture energy in Eq. (1) is defined on the assumption that both $\Gamma$ and $\theta$ at the current configuration are known. Standard computational fracture mechanics procedures revert to path tracking and optimization algorithms to predict and resolve the crack geometry as it evolves.

To avoid this, phase field approximation redefines the surface energy line integral expression into a representation over the entire domain $\Omega$ through the following approximation [11]

$$
\Psi_{f}=\int_{\Gamma} \mathcal{G}_{c}(\theta) d \Gamma \approx \int_{\Omega} \overline{\mathcal{G}}_{c} \mathcal{Z}_{c, A n i s} d \Omega
$$

where $\mathcal{Z}_{c, \text { Anis }}$ is a phase-field based functional defined over the entire domain $\Omega$. Parameter $\overline{\mathcal{G}}_{c}$ in Eq. (3) corresponds to the energy required to create a unit area of fracture surface $\mathcal{A}_{c}$. This multiplicative decomposition of the surface fracture energy effectively implies the following property for the anisotropic phase field functional, i.e.

$$
\mathcal{A}_{c}=\int_{\Omega} \mathcal{Z}_{c, \text { Anis }} d \Omega
$$


In this work, the 4 th order functional $\mathcal{Z}_{c, \text { Anis }}$ introduced in [21] is employed according to Eq. (4)

$$
\mathcal{Z}_{c, A n i s}=\left[\frac{(c-1)^{2}}{4 l_{0}}+l_{0}|\nabla c|^{2}+l_{0}^{3} \sum_{i j k l} \gamma_{i j k l} \frac{\partial^{2} c}{\partial x_{i} \partial x_{j}} \frac{\partial^{2} c}{\partial x_{k} \partial x_{l}}\right]
$$

where $c(\mathbf{x}, t) \in[0,1]$ is a phase-field defined over the domain $\Omega, l_{0} \in \mathbb{R}$ is a length scale parameter and $\gamma_{i j k l}, i, j, k, l=1 \ldots 3$ are the components of a $4^{\text {th }}$ order anisotropic tensor corresponding to the anisotropic constitutive behaviour of the material. The length scale parameter $l_{0}$ essentially defines a region in the vicinity of the crack path where the fracture energy is released. The larger the value of $l_{0}$ the larger the area over which the effect of fracture is effectively smeared. Values of $l_{0} \rightarrow 0$ provide solutions that converge to the discrete fracture solution [11].

Considering the limit value of phase field $c=1 \mathrm{in} \mathrm{Eq.} \mathrm{(4)} \mathrm{results} \mathrm{in} \mathrm{the}$ minimum value of the crack surface integral, i.e., $\Psi_{f}=0$ which corresponds to the uncracked state. Similarly, it can be shown that the limit value $c=0$ corresponds to the maximum value of $\Psi_{f}$ by asking that $\partial \mathcal{Z}_{c, A n i s} / \partial c$.

The tensor $\gamma$ is conveniently defined in the three dimensional space utilizing Voigt notation as

$$
\gamma=\left[\begin{array}{llllll}
\gamma_{1111} & \gamma_{1122} & \gamma_{1133} & \gamma_{1112} & \gamma_{1123} & \gamma_{1113} \\
\gamma_{2211} & \gamma_{2222} & \gamma_{2233} & \gamma_{2212} & \gamma_{2223} & \gamma_{2213} \\
\gamma_{3311} & \gamma_{3322} & \gamma_{3333} & \gamma_{3312} & \gamma_{3323} & \gamma_{3313} \\
\gamma_{1211} & \gamma_{1222} & \gamma_{1233} & \gamma_{1212} & \gamma_{1223} & \gamma_{1213} \\
\gamma_{2311} & \gamma_{2322} & \gamma_{2333} & \gamma_{2312} & \gamma_{2323} & \gamma_{2313} \\
\gamma_{1311} & \gamma_{1322} & \gamma_{1333} & \gamma_{1312} & \gamma_{1323} & \gamma_{1313}
\end{array}\right]
$$

whereas in the two-dimensional case $\gamma$ reduces to

$$
\gamma=\left[\begin{array}{lll}
\gamma_{1111} & \gamma_{1122} & \gamma_{1112} \\
\gamma_{2211} & \gamma_{2222} & \gamma_{2212} \\
\gamma_{1211} & \gamma_{1222} & \gamma_{1212}
\end{array}\right]
$$

Fracture propagation emerges form Eq. (3) through the minimization of the total potential energy; in addition, as a crack propagates within the domain $\Omega$ the value of the surface energy integral will be increasing with a corresponding decrease in the elastic strain energy.

This decrease corresponds to the degradation of material properties at the vicinity of the crack $\Gamma$ and is accounted for in phase field modelling by introducing a non-decreasing degradation function $g(c)$. This is superimposed on the positive part of the elastic strain energy density. Here, an additive decomposition of the energy density is implied into a purely tensile and a purely compressive part (see, e.g., [25]).

$$
\psi_{e l}=g(c) \psi_{e l}^{+}+\psi_{e l}^{-}
$$


where $\psi_{e l}^{+}$and $\psi_{e l}^{-}$correspond to the positive and negative parts of the elastic strain energy density and $g(c) \in[0,1]$. In this work, the degradation function introduced in [24] is utilized which assumes the following form

$$
g=(1-k) c^{2}+k
$$

where $0 \leq k \ll 1$ is a model parameter introduced in [2] to treat potential illconditioning. In this work the model parameter is considered to be $k=0$ with no impact on the stability of the resulting system of governing equations. It is evident from Eq. (7) that as $c \rightarrow 0$, i.e., when a crack evolves the degradation function decreases, thus reducing the overall positive part of elastic strain energy density in the deformable domain $\Omega$.

\subsection{Derivation of coupled strong form}

The strong form of the anisotropic brittle fracture problem is established herein by employing the methodology introduced in [9] for the purely isotropic case. The energy balance equation of the problem is defined in Eq. (9) as

$$
\dot{\mathcal{K}}(\dot{\mathbf{u}})+\dot{\mathcal{W}}^{\text {int }}(\dot{\mathbf{u}}, \dot{c}, \nabla \dot{c})-\dot{\mathcal{W}}^{e x t}(\dot{\mathbf{u}})=0
$$

where $\dot{\mathbf{u}}=d \mathbf{u} / d t$ corresponds to the velocity field, $\dot{c}=d c / d t$ is the phase field time derivative and $\nabla \dot{c}$ corresponds to the rate of the phase field spatial derivative, i.e.,

$$
\nabla \dot{c}=\frac{d}{d t}\left(\frac{\partial c}{\partial x_{i}}\right)
$$

for $i=1, \ldots, 3$.

The kinetic energy rate functional $\dot{\mathcal{K}}(\dot{\mathbf{u}})$ is evaluated as

$$
\dot{\mathcal{K}}(\dot{\mathbf{u}})=\frac{d}{d t} \int_{\Omega} \frac{1}{2} \rho|\dot{\mathbf{u}}|^{2} d \Omega
$$

where $\rho$ corresponds to the mass density and $\dot{\mathcal{W}}^{\text {ext }}(\dot{\mathbf{u}})$ is the rate of external work functional expressed as

$$
\dot{\mathcal{W}}^{e x t}(\dot{\mathbf{u}})=\int_{\partial \Omega_{\bar{t}}}(\overline{\boldsymbol{t}} \cdot \dot{\mathbf{u}}) d \partial \Omega_{\bar{t}}+\int_{\Omega}(\boldsymbol{b} \cdot \dot{\mathbf{u}}) d \Omega
$$

Finally, the rate of internal work functional $\dot{\mathcal{W}}^{\text {int }}(\dot{\mathbf{u}}, \dot{c}, \nabla \dot{c})$ is evaluated as

$$
\dot{\mathcal{W}}^{\text {int }}(\dot{\mathbf{u}}, \dot{c}, \nabla \dot{c})=\frac{d \Psi_{p o t}}{d t}=\frac{d}{d t} \int_{\Omega}\left(\psi_{e l}+\overline{\mathcal{G}}_{c} \mathcal{Z}_{c, A n i s}\right) d \Omega
$$


Applying the divergence theorem and performing the necessary algebraic manipulation, the balance of energy equation (9) becomes

$$
\begin{aligned}
& \int_{\partial \Omega_{\bar{t}}}([\boldsymbol{\sigma} \boldsymbol{n}-\overline{\boldsymbol{t}}] \cdot \dot{\mathbf{u}}) d \partial \Omega+\int_{\Omega}([\rho \ddot{\mathbf{u}}-\nabla \cdot \boldsymbol{\sigma}-\boldsymbol{b}] \cdot \dot{\mathbf{u}}) d \Omega+ \\
& \int_{\partial \Omega}\left(\left[\overline{\mathcal{G}}_{c} l_{0} 2 \nabla c-\overline{\mathcal{G}}_{c} l_{0}^{3} \sum_{i j k l} \gamma_{i j k l}\left(\frac{\partial^{3} c}{\partial x_{j} \partial x_{k} \partial x_{l}}\right)-\overline{\mathcal{G}}_{c} l_{0}^{3} \sum_{i j k l} \gamma_{i j k l}\left(\frac{\partial^{3} c}{\partial x_{i} \partial x_{j} \partial x_{k}}\right)\right] \cdot \boldsymbol{n} \dot{c}\right) d \partial \Omega+ \\
& \int_{\partial \Omega}\left(\left[\overline{\mathcal{G}}_{c} l_{0}^{3} \sum_{i j k l} \gamma_{i j k l}\left(\frac{d}{d t}\left(\frac{\partial c}{\partial x_{i}}\right) \frac{\partial^{2} c}{\partial x_{k} \partial x_{l}}\right)+\overline{\mathcal{G}}_{c} l_{0}^{3} \sum_{i j k l} \gamma_{i j k l}\left(\frac{\partial^{2} c}{\partial x_{i} \partial x_{j}} \frac{d}{d t}\left(\frac{\partial c}{\partial x_{l}}\right)\right)\right] \cdot \boldsymbol{n}\right) d \partial \Omega+ \\
& \int_{\Omega}\left[\psi_{e l_{c}}+\frac{(c-1)}{2 l_{0}}-\overline{\mathcal{G}}_{c} l_{0} 2 \Delta c+2 \overline{\mathcal{G}}_{c} l_{0}^{3} \sum_{i j k l} \gamma_{i j k l}\left(\frac{\partial^{4} c}{\partial x_{i} \partial x_{j} \partial x_{k} \partial x_{l}}\right)\right] \dot{c} d \Omega=0
\end{aligned}
$$

where $\boldsymbol{\sigma}$ is the stress field derived from the following relation

$$
\boldsymbol{\sigma}=\frac{\partial \psi_{e l}}{\partial \varepsilon}
$$

whereas $\ddot{\mathbf{u}}=d^{2} \mathbf{u} / d t^{2}$ is the acceleration field, $\boldsymbol{n}$ is the outward unit normal vector of the boundary and $\psi_{e l_{c}}=\partial \psi_{e l} / \partial c=2(1-k) c \psi_{e l}^{+}$is the derivative of elastic strain energy density with respect to the phase field $c$. The methodology employed for deriving Eq. (14) is presented in Appendix A.

Since Eq. (14) must hold for arbitrary values of $\dot{\mathbf{u}}$ and $\dot{c}$, the resulting strong form is eventually derived as

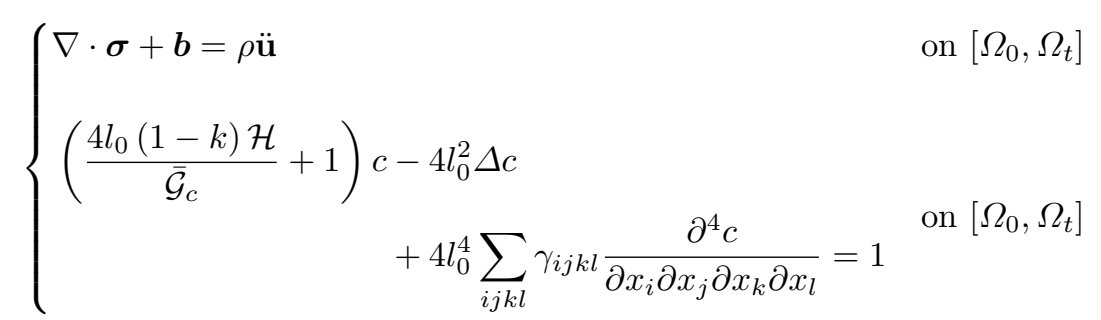

where $\mathcal{H}$ is the history field defined as the maximum value of the tensile part of the elastic energy density $\psi_{e l}^{+}$obtained in the domain $[0, t]$. 
The coupled field equations (16) are subject to the set of boundary and initial conditions presented in Eq. (17)

$$
\begin{cases}\boldsymbol{\sigma} \boldsymbol{n}=\overline{\boldsymbol{t}}, & \text { on }\left[\partial \Omega_{\bar{t}_{0}}, \partial \Omega_{\bar{t}_{t}}\right] \\ \mathbf{u}=\overline{\mathbf{u}}, & \text { on }\left[\partial \Omega_{\bar{u}_{0}}, \partial \Omega_{\bar{u}_{t}}\right] \\ \mathbf{u}=\mathbf{u}_{0}, & \text { on } \Omega_{0} \\ \dot{\mathbf{u}}_{0}=\dot{\mathbf{u}}_{0}, & \text { on } \Omega_{0} \\ \ddot{\mathbf{u}}=\ddot{\mathbf{u}}_{0}, & \text { on } \Omega_{0} \\ 4 l_{0}^{2} \nabla c-2 l_{0}^{4} \sum_{i j k l} \gamma_{i j k l}\left(\frac{\partial^{3} c}{\partial x_{j} \partial x_{k} \partial x_{l}}\right)-2 l_{0}^{4} \sum_{i j k l} \gamma_{i j k l}\left(\frac{\partial^{3} c}{\partial x_{i} \partial x_{j} \partial x_{k}}\right)=0, & \text { on }\left[\partial \Omega_{t_{0}}, \partial \Omega_{t}\right] \\ 2 l_{0}^{4} \sum_{i j k l} \gamma_{i j k l}\left(\frac{\partial^{2} c}{\partial x_{k} \partial x_{l}}\right)+2 l_{0}^{4} \sum_{i j k l} \gamma_{i j k l}\left(\frac{\partial^{2} c}{\partial x_{i} \partial x_{j}}\right)=0, & \text { on }\left[\partial \Omega_{t_{0}}, \partial \Omega_{t}\right] \\ c=c_{0}, & \text { on } \Omega_{0}\end{cases}
$$

where $\mathbf{u}_{0}, \dot{\mathbf{u}}_{0}, \ddot{\mathbf{u}}_{0}$ and $c_{0}$ are the initial conditions for the displacement, velocity, acceleration and phase field respectively. Furthermore, $\Omega_{0}, \partial \Omega_{\bar{t}_{0}}, \partial \Omega_{\bar{u}_{0}}$ correspond to the initial domain volume, traction surface and constrained surfaces respectively whereas $\partial \Omega_{\bar{t}_{t}}, \partial \Omega_{\bar{u}_{t}}$ correspond to the traction and constrained surfaces at time $t$.

\subsection{Elastic Energy density decomposition}

The decomposition of the elastic strain energy density into a positive (due to tension) $\psi_{e l}^{+}$and negative (due to compression) $\psi_{e l}^{-}$(see Eq. (7)) is an integral aspect of phase field methodology employed to essentially couple the evolution of phase field $c$ to the evolution of tensile stresses within the domain $\Omega$. During the past few years, various methodologies have been introduced to address this energy split; a detailed review of the existing models can be retrieved in [22].

In this work, we adopt the elastic strain energy density decomposition for isotropic materials introduced in [25]. According to this approach, the tensile and negative components are defined as

$$
\psi_{e l}^{+}=\frac{1}{2} \lambda\langle\operatorname{Tr}[\varepsilon]\rangle^{+^{2}}+\mu \operatorname{Tr}\left[\left(\varepsilon^{+}\right)^{2}\right]
$$

and

$$
\psi_{e l}^{-}=\frac{1}{2} \lambda\langle\operatorname{Tr}[\varepsilon]\rangle^{-^{2}}+\mu \operatorname{Tr}\left[\left(\varepsilon^{-}\right)^{2}\right]
$$


respectively, where $\lambda$ and $\mu$ are the Lamé constants and $\varepsilon$ corresponds to the strain tensor. The symbols $\langle.\rangle^{+}$and $\langle.\rangle^{-}$are defined as $\langle x\rangle^{+}=(x+|x|) / 2$ and $\langle x\rangle^{-}=(x-|x|) / 2$ respectively, whereas the symbol Tr stands for the trace. The positive part of the strain tensor $\varepsilon^{+}$in Eq. (18) is defined through the following spectral decomposition

$$
\varepsilon^{+}=\boldsymbol{P} \boldsymbol{\Lambda}^{+} \boldsymbol{P}^{T}
$$

where $\boldsymbol{P}$ is a matrix whose columns comprise the eigen-vectors of the strain tensor $\varepsilon$ and $\boldsymbol{\Lambda}^{+}$is a diagonal matrix defined as

$$
\boldsymbol{\Lambda}^{+}=\operatorname{diag}\left(\left\langle\lambda_{1}\right\rangle^{+},\left\langle\lambda_{2}\right\rangle^{+},\left\langle\lambda_{3}\right\rangle^{+}\right)
$$

where $\lambda_{i}, i=1, \ldots, 3$ are the eigen-values of the strain tensor. The negative part of strain tensor in relation (19) is evaluated as

$$
\varepsilon^{-}=\varepsilon-\varepsilon^{+}
$$

In view of the definition of the decomposition of the elastic energy density (Eq. (7)), the corresponding analytical expressions defined in Eqs. (18) and (19) and the stress field in Eq. (15) the material constitutive tangent stiffness matrix is readily derived from equation

$$
\boldsymbol{D}=\frac{\partial \boldsymbol{\sigma}}{\partial \varepsilon}
$$

2.4 Special cases of anisotropy in surface energy density

In this work, the cases of both cubic and orthotropic symmetry are considered for the surface energy density. In the case of cubic symmetry the 4-th order tensor $\gamma$ introduced in Eq. (5) is expressed in the material principal axes, as

$$
\gamma=\left[\begin{array}{cccccc}
\gamma_{11} & \gamma_{12} & \gamma_{12} & 0 & 0 & 0 \\
\gamma_{12} & \gamma_{11} & \gamma_{12} & 0 & 0 & 0 \\
\gamma_{12} & \gamma_{12} & \gamma_{11} & 0 & 0 & 0 \\
0 & 0 & 0 & 4 \gamma_{44} & 0 & 0 \\
0 & 0 & 0 & 0 & 4 \gamma_{44} & 0 \\
0 & 0 & 0 & 0 & 0 & 4 \gamma_{44}
\end{array}\right]
$$

Voigt notation is employed in Eq. (24) for brevity [40]. Similarly, in the case of orthotropic symmetry the $\gamma$ assumes the following form

$$
\gamma=\left[\begin{array}{cccccc}
\gamma_{11} & \gamma_{12} & \gamma_{13} & 0 & 0 & 0 \\
\gamma_{12} & \gamma_{22} & \gamma_{23} & 0 & 0 & 0 \\
\gamma_{13} & \gamma_{33} & \gamma_{33} & 0 & 0 & 0 \\
0 & 0 & 0 & 4 \gamma_{44} & 0 & 0 \\
0 & 0 & 0 & 0 & 4 \gamma_{55} & 0 \\
0 & 0 & 0 & 0 & 0 & 4 \gamma_{66}
\end{array}\right]
$$

In Section 4 examples are presented for both cases and the corresponding responses are compared and discussed. 


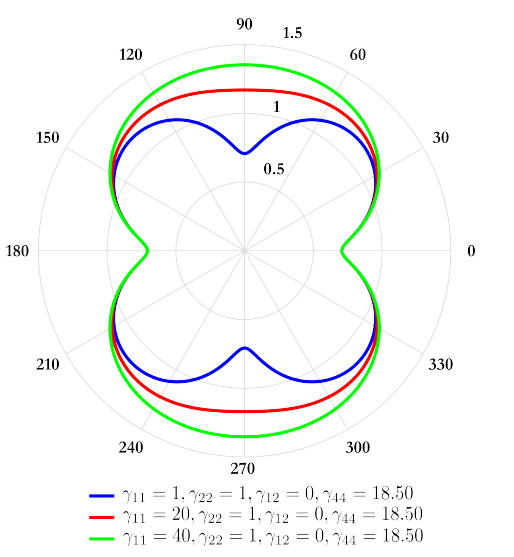

(a)

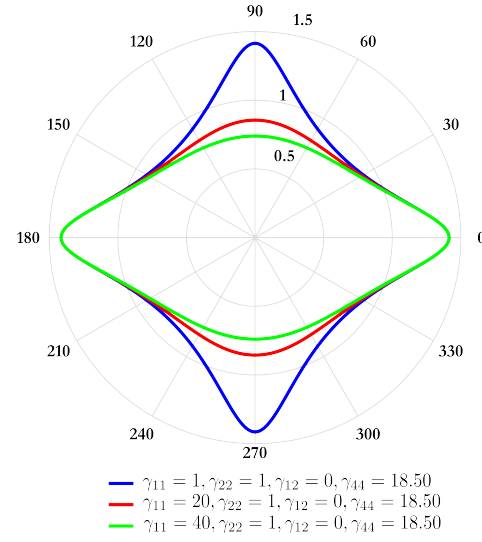

(b)

Fig. 2 Polar plots of (a) surface energy density $\mathcal{G}_{c}(\theta)$ and (b) reciprocal of surface energy density $1 / \mathcal{G}_{c}(\theta)$ for increasing values of component $\gamma_{11}$

\subsection{Influence of anisotropic parameters in surface energy density}

To further highlight the assumed anisotropic material behaviour with respect to fracture, the form of the surface energy density $\mathcal{G}_{c}(\theta)$ as a function of the fracture orientation $\theta$ is investigated in this section for characteristic cases of anisotropy. Detailed derivations of the surface energy plots presented herein is provided in Appendix B.

The two-dimensional case is considered herein for brevity, in which case the anisotropic tensor $\gamma$ (see equation (25)) reduces to

$$
\gamma=\left[\begin{array}{ccc}
\gamma_{11} & \gamma_{12} & 0 \\
\gamma_{12} & \gamma_{22} & 0 \\
0 & 0 & 4 \gamma_{44}
\end{array}\right]
$$

In Fig. 2, the distribution of the surface energy density $\mathcal{G}_{c}(\theta)$ and its reciprocal $1 / \mathcal{G}_{c}(\theta)$ is shown for the case of increasing values of component $\gamma_{11}$ while all other components are kept constant. When $\gamma_{11}=\gamma_{22}$ the case of four-fold symmetry, i.e., cubic symmetry is manifested whereas in the case of $\gamma_{11} \neq \gamma_{22}$ the case of two-fold symmetry, i.e., orthotropic symmetry is revealed. Increasing values of component $\gamma_{11}$ result in increasing surface energy density values along the $90 \mathrm{deg}$ and $270 \mathrm{deg}$ fracture directions.

Similarly, increasing values of component $\gamma_{22}$ when all other components of the anisotropic fracture tensor are kept constant results in increasing values of the surface energy density along the $0 \mathrm{deg}$ and $180 \mathrm{deg}$ directions of fracture as shown in Fig. 3 .

Thus, the values of the normal components of the anisotropic tensor $\gamma$ control the extreme values of the corresponding surface energy density. The effect of the shear components of the anisotropic tensor, i.e., $\gamma_{12}$ and $\gamma_{44}$ is 


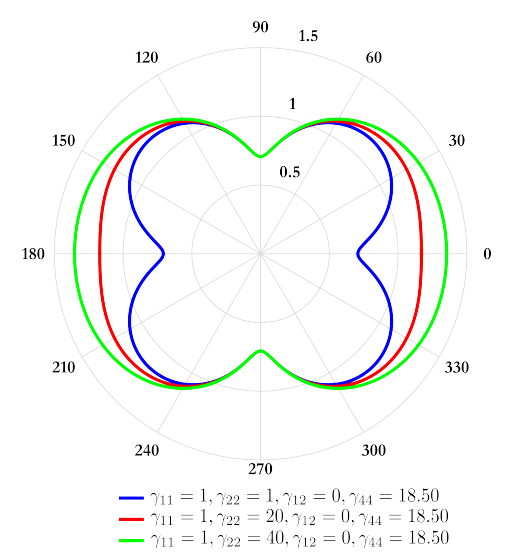

(a)

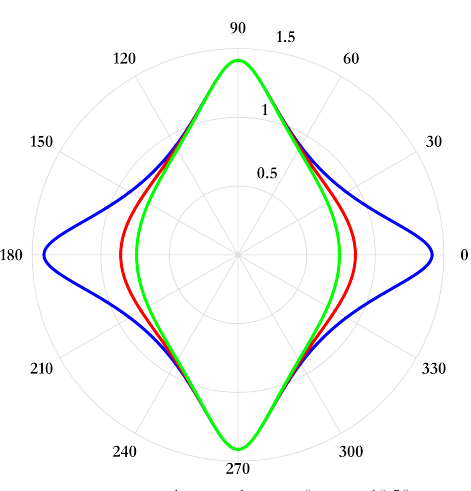

$\gamma_{11}=1, \gamma_{22}=1, \gamma_{12}=0, \gamma_{44}=18.50$
$\gamma_{11}=1, \gamma_{22}=20, \gamma_{12}=0, \gamma_{44}=18.50$ $\gamma_{11}=1, \gamma_{22}=20, \gamma_{12}=0, \gamma_{44}=18.50$
$-\gamma_{11}=1, \gamma_{22}=40, \gamma_{12}=0, \gamma_{44}=18.50$

(b)

Fig. 3 Polar plots of (a) surface energy density $\mathcal{G}_{c}(\theta)$ and (b) reciprocal of surface energy density $1 / \mathcal{G}_{c}(\theta)$ for increasing values of component $\gamma_{22}$

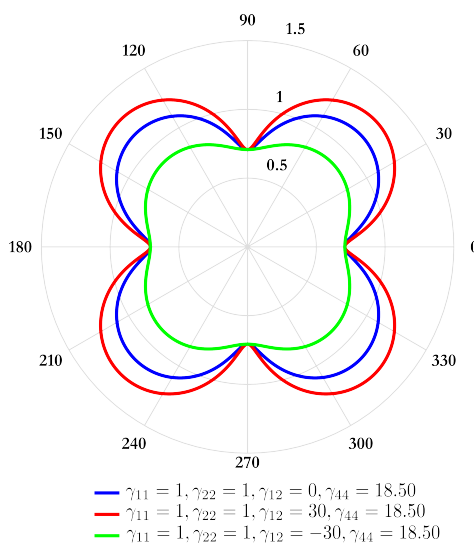

(a)

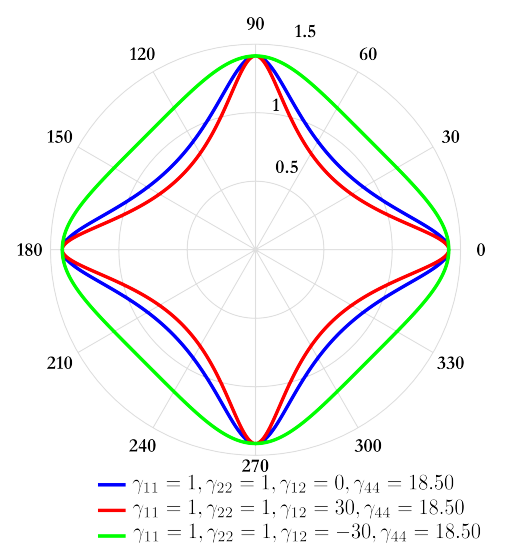

(b)

Fig. 4 Polar plots of (a) surface energy density $\mathcal{G}_{c}(\theta)$ and (b) reciprocal of surface energy density $1 / \mathcal{G}_{c}(\theta)$ for increasing values of component $\gamma_{12}$

illustrated in Figs. 4 and 5 respectively. Both parameters control the extreme values of both the surface energy density and its reciprocal at a rotated coordinate system with respect to the origin. 


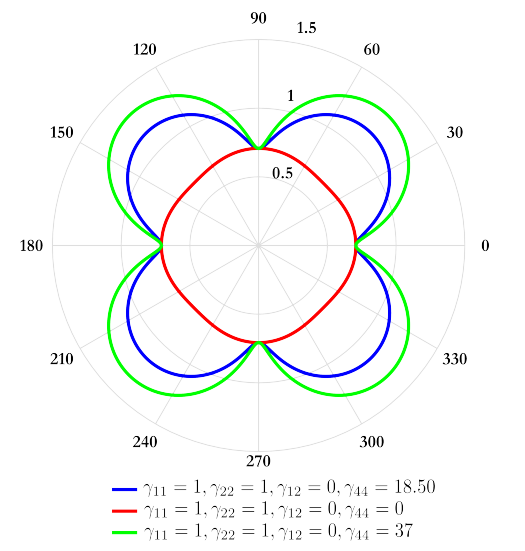

(a)

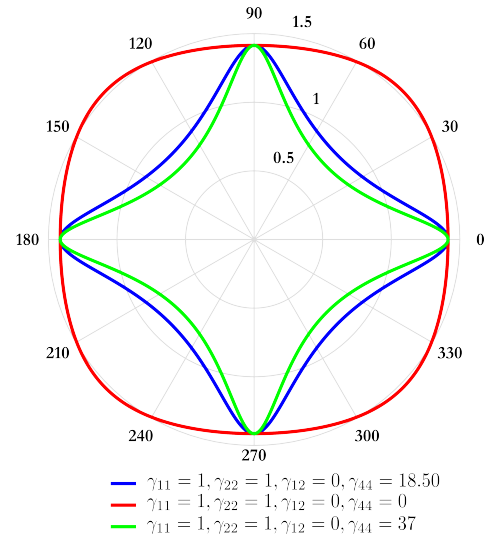

(b)

Fig. 5 Polar plots of (a) surface energy density $\mathcal{G}_{c}(\theta)$ and (b) reciprocal of surface energy density $1 / \mathcal{G}_{c}(\theta)$ for increasing values of component $\gamma_{44}$

\section{Numerical Implementation}

\subsection{Material Point Method approximation}

MPM approximation is based on the discretization of a deformable domain $\Omega$ with a set of material points (Fig. 6). These can be defined arbitrarily as collocation points within any appropriate tessellation of the domain $\Omega$. In this work, initial positions of material points are chosen to coincide with the Gauss points of the corresponding standard finite element discretization, both for brevity but also to facilitate comparisons with the standard FEM. In Fig. 6 , the domain $\Omega$ is discretized into a set $\mathcal{P}=\left\{p \mid p=1,2, \ldots, N_{p}\right\}$, where $N_{p} \in \mathbb{Z}$ is the total number of material points and subscript $p$ indexes the $p^{t h}$ material point.

Based on this discretization, both the mass density $\rho$ and domain volume $\Omega$ are additively decomposed into the corresponding material point contributions, i.e,

$$
\rho(\mathbf{x}, t)=\sum_{p=1}^{N_{p}} \rho_{p} \delta\left(\mathbf{x}-\mathbf{x}_{p_{t}}\right)
$$

and

$$
\Omega(\mathbf{x}, t)=\sum_{p=1}^{N_{p}} \Omega_{p} \delta\left(\mathbf{x}-\mathbf{x}_{p_{t}}\right)
$$

respectively. In Eqs. (26) and (27) above, $\rho_{p}=M_{p} / \Omega_{p}$ is the mass density of the material point, $M_{p}$ is the material point mass, $\Omega_{p}$ is the material point volume and $\delta$ is the Dirac function.

Kinematics of the material points are introduced in a straightforward manner following the Lagrangian description of continuum mechanics [6]. Under an 


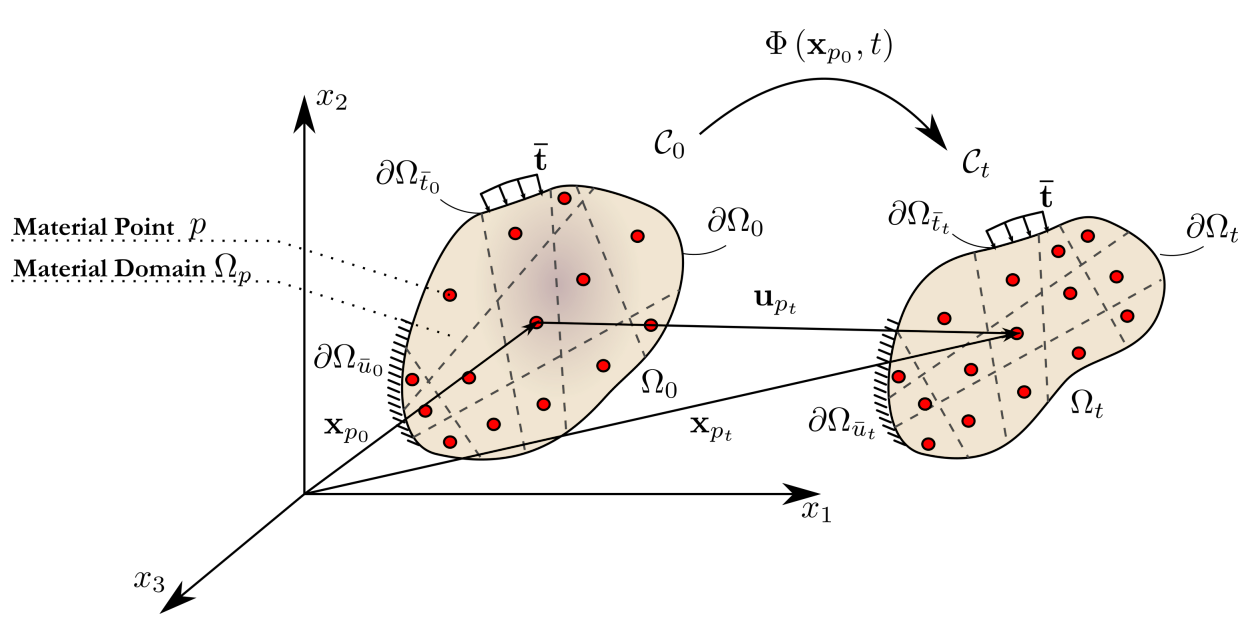

Fig. 6 Deformable domain discretization into material points

arbitrary motion $\Phi\left(\mathbf{x}_{p_{0}}, t\right)$ an initial material configuration $\mathcal{C}_{0}$ is transformed onto the current configuration $\mathcal{C}_{t}$. The displacement of each material point is defined as $\mathbf{u}_{p_{t}}=\mathbf{x}_{p_{t}}-\mathbf{x}_{p_{0}}$ where $\mathbf{x}_{p_{0}}$ and $\mathbf{x}_{p_{t}}$ are the position vectors of the $p^{t h}$ material point respectively.

Further to the material point discretization, MPM introduces a finite element grid, termed the background or Eulerian grid (Fig. 7). This is a nondeforming mesh where material point response quantities, e.g., displacements are mapped into and solution of the governing equations is performed. The Eulerian grid is essentially the computational space through which material points are moving. At any given time instant $t$, the background grid comprises the set of active cells, i.e., cells where at a material point resides and the inactive cells where no material point exists. In this work, the background grid is constantly updated according to the topology of the material points, thus reducing the solution space at any time instant.

Mapping of the material point response quantities into the nodes of the corresponding background active cell is performed by means of the finite element interpolation functions pertinent to this active cell. In this work, the background grid is defined using a structured mesh of quadrilateral plain stress/ strain elements employing higher-order B-Splines as interpolation functions. The use of higher-order B-splines is necessitated by the 4th order phase field functional utilized in this work (see also Section 2) but also results in an efficient treatment of cell crossing errors pertinent to MPM [36]. 


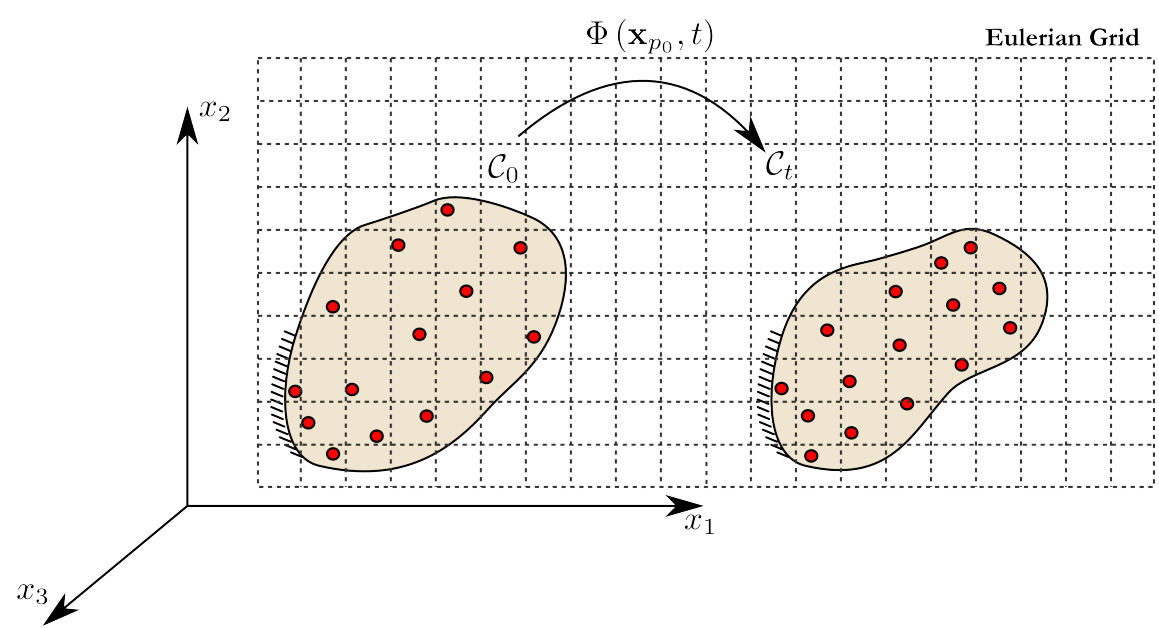

Fig. 7 Material Point Method Eulerian Grid

\subsubsection{B-spline interpolation}

B-Splines are defined through a non-decreasing sequence of real numbers (i.e. $\left.\xi_{I} \leq \xi_{I+1}\right)$, termed the knot vectors, and formally described as

$$
\Xi=\left\{\xi_{1}, \xi_{2}, \ldots, \xi_{N_{n_{\xi}}+q_{\xi}+1}\right\}
$$

where $N_{n_{\xi}}$ and $q_{\xi}$ are the number of basis functions and the polynomial order in the $\xi$ direction respectively. The $I^{t h} \mathrm{~B}$-spline basis function of $q_{\xi}$-degree is defined recursively as

$$
N_{I, q_{\xi}}(\xi)=\frac{\xi-\xi_{I}}{\xi_{I+q_{\xi}}-\xi_{I}} N_{I, q_{\xi}-1}(\xi)+\frac{\xi_{I+q_{\xi}+1}-\xi}{\xi_{I+q_{\xi}+1}-\xi_{I+1}} N_{I+1, q_{\xi}-1}(\xi)
$$

where

$$
N_{I, 0}(\xi)= \begin{cases}1 & \xi_{I} \leq \xi \leq \xi_{I+1} \\ 0 & \text { otherwise }\end{cases}
$$

Furthermore, the derivative of a B-Spline basis function is expressed as

$$
N_{I, q_{\xi}}^{\prime}(\xi)=\frac{q_{\xi}}{\xi_{i+q_{\xi}}-\xi_{i}} N_{I, q_{\xi}-1}(\xi)-\frac{q_{\xi}}{\xi_{i+q_{\xi}+1}-\xi_{i+1}} N_{I+1, q_{\xi}-1}(\xi)
$$

Higher order derivatives are evaluated recursively from relation (31). A BSpline curve is evaluated as

$$
\mathbf{C}(\xi)=\sum_{I=1}^{N_{n_{\xi}}} N_{I, q_{\xi}}(\xi) \mathbf{P}_{I}
$$

where $\mathbf{P}_{I} \in \mathbb{R}_{d}, I=1,2, . ., N_{n_{\xi}}$ are the coordinates of control points. $d$ is the dimension of the problem. 


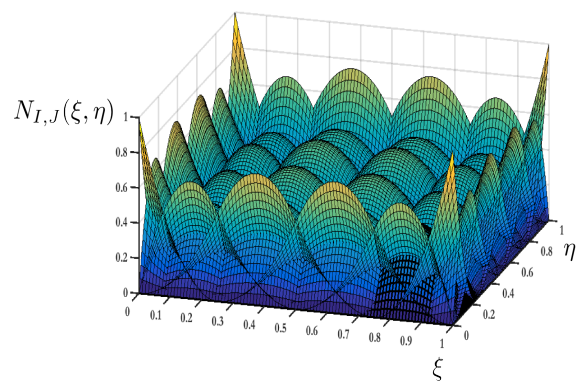

(a)

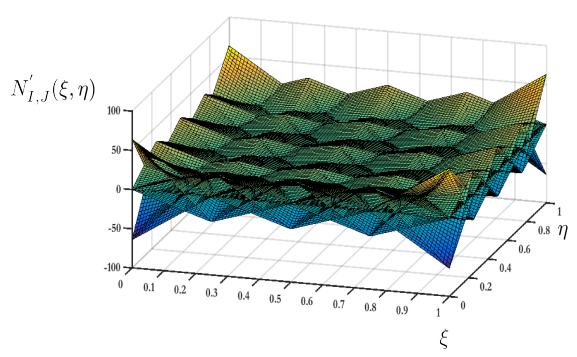

(b)

Fig. 8 High-Order B-Splines: (a) Quadratic basis functions $\left(C^{1}\right)$ and (b) their first derivatives

The two-dimensional quadratic basis functions $\left(\left(q_{\xi}=q_{\eta}=2\right)\right)$ together with their first derivatives are shown in Fig. 8 for knot vectors $\Xi=H=$ $\{0,0,0,0.25,0.50,0.75,1,1,1\}$.

\subsubsection{Material Point Method computational cycle}

The overall computational cycle of the material point method is based on the incremental exchange of information between the Lagrangian and Eulerian components of the method. In each incremental step, the updated material point stress components are mapped onto the background mesh nodes using the B-spline interpolation functions described in Section 3.1.1.

Next, solution is performed either in an explicit or implicit fashion and the resulting updated displacement components are mapped back onto the material points to identify their new state in terms of displacements, strains and stresses. At the end of the computational cycle, the background mesh is reset to its initial position. This is a crucial step within the MPM setting as distortion of the solution space is avoided thus resulting in a high-fidelity computational procedure for complex phenomena (see, e.g., $[19,5,17]$ ). The MPM computational cycle within an incremental step is summarized in Fig. 9. Further information on the numerical scheme can be found in $[37,38]$.

\subsection{Discrete equilibrium equations}

The discrete form of the MPM equilibrium equations is derived in a straightforward manner by means of the Galerkin approximation [6]. In this work only quasi-static problems are considered. The weak form of the equilibrium equations introduced in the first of Eqs. (16) is expressed as

$$
\int_{\Omega}(\boldsymbol{\sigma}: \nabla \boldsymbol{w}) d \Omega=\int_{\partial \Omega_{\bar{t}}}(\overline{\boldsymbol{t}} \cdot \boldsymbol{w}) d \partial \Omega_{\bar{t}}+\int_{\Omega}(\boldsymbol{b} \cdot \boldsymbol{w}) d \Omega
$$




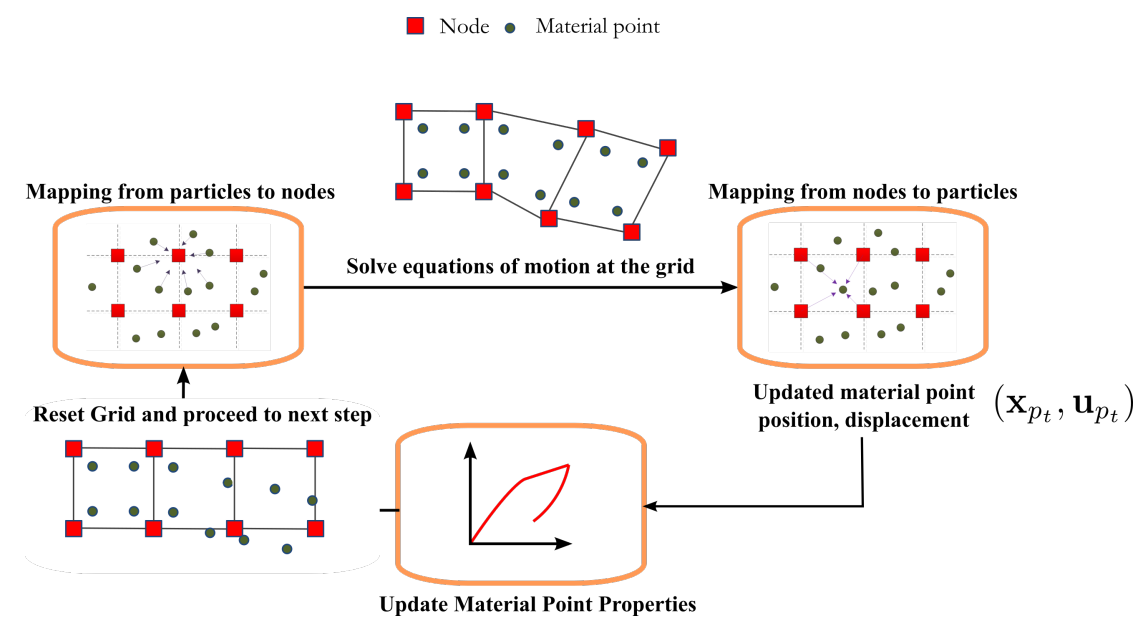

Fig. 9 Material Point Method computational cycle

where the following solution space is defined for the weighting functions $\boldsymbol{w}$.

$$
\mathcal{D}=\left\{\boldsymbol{w} \in H_{1}(\Omega) \quad \mid \boldsymbol{w}=0 \text { on } \partial \Omega_{\bar{u}}\right\}
$$

Similarly, the following Hilbert space is considered for the trial functions of the displacement field, i.e.,

$$
\mathcal{V}=\left\{\mathbf{u} \in H_{1}(\Omega) \quad \mid \mathbf{u}=\overline{\mathbf{u}} \text { on } \partial \Omega_{\bar{u}}\right\}
$$

Substituting for the material point approximation introduced in equations (26) and (27) the following discrete equation is established

$$
\sum_{p=1}^{N_{p}}\left(\boldsymbol{\sigma}_{p}: \boldsymbol{\nabla} \boldsymbol{w}_{p}\right) \Omega_{p}=\int_{\partial \Omega_{\bar{t}}}(\overline{\boldsymbol{t}} \cdot \boldsymbol{w}) d \partial \Omega_{\bar{t}}+\sum_{p=1}^{N_{p}}\left(\boldsymbol{b}_{p} \cdot \boldsymbol{w}_{p}\right) \Omega_{p}
$$

where $\boldsymbol{w}_{p}, \boldsymbol{\sigma}_{p}$ and $\boldsymbol{b}_{p}$ are the test functions, stress field and body forces evaluated at each material point $\mathbf{x}_{p}$.

The test functions $\boldsymbol{w}_{p}$ and their spatial derivatives $\boldsymbol{\nabla} \boldsymbol{w}_{p}$ are interpolated in the Galerkin sense according to relations (35)

$$
\boldsymbol{w}_{p}=\sum_{I=1}^{N_{n}} N_{I}\left(\mathbf{x}_{p}\right) \boldsymbol{w}_{I}
$$

and (36) respectively

$$
\boldsymbol{\nabla} \boldsymbol{w}_{p}=\sum_{I=1}^{N_{n}} \nabla N_{I}\left(\mathbf{x}_{p}\right) \boldsymbol{w}_{I}
$$

where $N_{n}$ is the number of grid nodes and $N_{I}\left(\mathbf{x}_{p}\right)$ are the higher-order B-spline interpolation functions (Eq. (29)) evaluated at the material points. Furthermore, $\boldsymbol{w}_{I}$ are the test function nodal values whereas $I$ refers to the $I^{\text {th }}$ grid node. Similar expressions hold for the displacement test functions. 
Substituting in relation (34), considering equation (23) and performing the necessary algebraic manipulation, the following set of discrete equilibrium equations is finally established

$$
\boldsymbol{R}_{I}^{u}\left(\mathbf{u}_{I}\right)=\boldsymbol{F}_{I}^{i n t}-\boldsymbol{F}_{I}^{e x t}=0, \quad I=1 \ldots, N_{n}
$$

where $\boldsymbol{F}_{I}^{\text {int }}$ is the vector of corresponding internal forces defined as

$$
\boldsymbol{F}_{I}^{i n t}=\sum_{p=1}^{N_{p}}\left(\boldsymbol{\sigma}_{p} \cdot \nabla N_{I}\left(\mathbf{x}_{p}\right)\right) \Omega_{p}
$$

and $\boldsymbol{F}_{I}^{e x t}$ corresponds for the equivalent vector of external forces evaluated at grid node $I$ expressed as

$$
\boldsymbol{F}_{I}^{e x t}=\int_{\partial \Omega_{\bar{t}}}\left(\overline{\boldsymbol{t}} N_{I}\left(\mathbf{x}_{p}\right)\right) d \partial \Omega_{\bar{t}}+\sum_{p=1}^{N_{p}} \boldsymbol{b}_{p} N_{I}\left(\mathbf{x}_{p}\right) \Omega_{p}
$$

The residual nodal values $\boldsymbol{R}_{I}^{u}$ are expressed as a function of the discrete displacement field $\mathbf{u}_{I}$.

\subsection{Discrete anisotropic phase field equations}

The discrete form of the anisotropic phase field governing equations introduced in the second of Eqs. (16) is derived accordingly. In particular, the phase field weak introduced in the second of Eqs. (16) becomes

$$
\begin{aligned}
\int_{\Omega}\left(\frac{4 l_{0}(1-k) \mathcal{H}}{\overline{\mathcal{G}}_{c}}+1\right) c q d \Omega & +\int_{\Omega} 4 l_{0}^{2}(\nabla c: \nabla q) d \Omega \\
& +\int_{\Omega} 4 l_{0}^{4} \sum_{i j k l} \gamma_{i j k l}\left(\frac{\partial^{2} c}{\partial x_{i} \partial x_{j}} \frac{\partial^{2} q}{\partial x_{k} \partial x_{l}}\right) d \Omega \\
& =\int_{\Omega} q d \Omega
\end{aligned}
$$

where $c$ is the phase field and $q$ are the corresponding weighting functions. The phase field $c$ and the corresponding weighting functions $q$ are defined with respect to the following spaces, i.e.,

$$
\mathcal{Y}=\left\{c \in H_{2}(\Omega)\right\}
$$

and

$$
\mathcal{Q}=\left\{q \in H_{2}(\Omega)\right\}
$$


Introducing the MPM approximation (Eq. (27)) into Eq. (40), the following expression is retrieved

$$
\begin{aligned}
\sum_{p=1}^{N_{p}} \mathcal{F}_{p} c_{p} q_{p} \Omega_{p} & +\sum_{p=1}^{N_{p}} 4 l_{0_{p}}^{2}\left(\nabla c_{p}: \nabla q_{p}\right) \Omega_{p} \\
& +\sum_{p=1}^{N_{p}} 4 l_{0_{p}}^{4} \sum_{i j k l} \gamma_{i j k l_{p}}\left(\frac{\partial^{2} c_{p}}{\partial x_{i} \partial x_{j}} \frac{\partial^{2} q_{p}}{\partial x_{k} \partial x_{l}}\right) \Omega_{p}=\sum_{p=1}^{N_{p}} q_{p} \Omega_{p}
\end{aligned}
$$

where $c_{p}, q_{p}$ and $\gamma_{i j k l_{p}}$ are the phase field, weighting functions and anisotropic tensor components evaluated at the material point $p$. Parameter $\mathcal{F}_{p}$ in Eq. (41) is expressed as

$$
\mathcal{F}_{p}=\frac{4 l_{0_{p}}\left(1-k_{p}\right) \mathcal{H}_{p}}{\overline{\mathcal{G}}_{c_{p}}}+1
$$

where $l_{0_{p}}, k_{p}, \mathcal{H}_{p}$ and $\overline{\mathcal{G}}_{c_{p}}$ are the length scale parameter, model parameter, history field and critical fracture energy density of material point $\mathbf{x}_{p}$.

Both $c_{p}$ and $q_{p}$ are interpolated at the nodal points of the background mesh, using the higher-order B-spline interpolation functions as in the case of the displacement field. Use of B-spline functions is required in this case, as second derivatives of the phase field appear on the variational expression (40). Thus, the interpolation relations considered assume the following form for the test functions and their first and second derivatives, i.e.,

$$
\begin{gathered}
q_{p}=\sum_{I=1}^{N_{n}} N_{I}\left(\mathbf{x}_{p}\right) q_{I} \\
\nabla q_{p}=\sum_{I=1}^{N_{n}} \nabla N_{I}\left(\mathbf{x}_{p}\right) q_{I}
\end{gathered}
$$

and

$$
\Delta q_{p}=\sum_{I=1}^{N_{n}} \Delta N_{I}\left(\mathbf{x}_{p}\right) q_{I}
$$

respectively. In Eqs. (43)-(45), $N_{I}\left(\mathbf{x}_{p}\right)$ are the background mesh shape functions pertinent to the phase field interpolation and $q_{I}$ are nodal values of the corresponding test functions.

Similarly, the material point phase field is interpolated at the nodal values of its parent cell $c_{I}$ according to Eq. (46)

$$
c_{p}=\sum_{I=1}^{N_{n}} N_{I}\left(\mathbf{x}_{p}\right) c_{I}
$$

whereas a similar interpolation scheme is assumed for its first

$$
\nabla c_{p}=\sum_{I=1}^{N_{n}} \nabla N_{I}\left(\mathbf{x}_{p}\right) c_{I}
$$


and second derivative

$$
\Delta c_{p}=\sum_{I=1}^{N_{n}} \Delta N_{I}\left(\mathbf{x}_{p}\right) c_{I}
$$

respectively.

Substituting, Eqs. (43),(44) and (45) into the discrete expression (41), the following relation is established

$$
R_{I}^{c}\left(c_{I}\right)={ }^{(I)} \mathcal{S}_{1}+{ }^{(I)} \mathcal{S}_{2}+{ }^{(I)} \mathcal{S}_{3}-{ }^{(I)} \mathcal{S}_{4}=0, \quad I=1 \ldots, N_{n}
$$

where

$$
\begin{gathered}
{ }^{(I)} \mathcal{S}_{1}=\sum_{p=1}^{N_{p}} \mathcal{F}_{p} c_{p} N_{I}\left(\mathbf{x}_{p}\right) \Omega_{p}, \\
{ }^{(I)} \mathcal{S}_{2}=\sum_{p=1}^{N_{p}} 4 l_{0_{p}}^{2}\left(\nabla c_{p} \cdot \nabla N_{I}\left(\mathbf{x}_{p}\right)\right) \Omega_{p}, \\
{ }^{(I)} \mathcal{S}_{3}=\sum_{p=1}^{N_{p}} 4 l_{0_{p}}^{4} \sum_{i j k l} \gamma_{i j k l_{p}}\left(\frac{\partial^{2} c_{p}}{\partial x_{i} \partial x_{j}} \frac{\partial^{2} N_{I}\left(\mathbf{x}_{p}\right)}{\partial x_{k} \partial x_{l}}\right) \Omega_{p},
\end{gathered}
$$

and

$$
{ }^{(I)} \mathcal{S}_{4}=\sum_{p=1}^{N_{p}} N_{I}\left(\mathbf{x}_{p}\right) \Omega_{p}
$$

respectively whereas $R_{I}^{c}\left(c_{I}\right)$ denotes the residual nodal values for the phase field.

Furthermore, introducing the phase field interpolation schemes (Eqs. (46) to (48)) and performing the necessary algebraic manipulation, the material point discrete phase field equations for the case of anisotropic fracture are cast in the following convenient form

$$
\boldsymbol{K}^{c} \boldsymbol{c}=\boldsymbol{F}^{c}
$$

where $\boldsymbol{K}^{c}$ is an $\left(N_{n} \times N_{n}\right)$ coefficient matrix whose $K_{I, J}^{c}$ component is expressed as

$$
\begin{aligned}
K_{I, J}^{c}=\sum_{p=1}^{N_{p}}\left(\mathcal{F}_{p} N_{J}\left(\mathbf{x}_{p}\right) N_{I}\left(\mathbf{x}_{p}\right)\right. & +4 l_{0_{p}}^{2}\left(\nabla N_{J}\left(\mathbf{x}_{p}\right) \cdot \nabla N_{I}\left(\mathbf{x}_{p}\right)\right) \\
& \left.+4 l_{0_{p}}^{4} \sum_{i j k l} \gamma_{i j k l_{p}}\left(\frac{\partial^{2} N_{J}\left(\mathbf{x}_{p}\right)}{\partial x_{i} \partial x_{j}} \frac{\partial^{2} N_{I}\left(\mathbf{x}_{p}\right)}{\partial x_{k} \partial x_{l}}\right)\right) \Omega_{p}
\end{aligned}
$$

Vector $\boldsymbol{c}$ is the $\left(N_{n} \times 1\right)$ vector of unknown nodal phase fields and $\boldsymbol{F}^{c}$ is the $\left(N_{n} \times 1\right)$ vector whose $F_{I}^{c}$ component is defined as

$$
F_{I}^{c}=\sum_{p=1}^{N_{p}} N_{I}\left(\mathbf{x}_{p}\right) \Omega_{p}
$$

The vector quantity $\boldsymbol{F}^{c}$ will be termed herein as the phase field forcing term. 


\subsection{Phase field Material Point Method solution scheme}

In this work, a staggered iterative scheme [25] is adopted for the solution of the system of coupled discrete equations (37) and (54). In this, the phase field equations are initially solved for the current value of the history field $\mathcal{H}$, and a prediction for the phase field nodal values $c_{I}$ is established. Next, the phase field nodal values $c_{I}$ are mapped to each material point and the degradation function $g_{p}$ is evaluated at each material point. Using the current value of the degradation function $g_{p}$, the equilibrium equations are iteratively solved within a Newton-Raphson scheme $\left(j=1,2, \ldots, N_{\text {iters }}\right)$ and the incremental nodal displacements $\Delta \mathbf{u}_{I}$ are derived. Within a displacement control setting, Eq. (37) is reformulated as

$$
\delta \boldsymbol{R}_{I}^{u}\left(\boldsymbol{\Delta} \mathbf{u}_{I}\right)=\boldsymbol{\Delta} \boldsymbol{F}_{I}^{i n t}-\boldsymbol{\Delta} \boldsymbol{F}_{I}^{e x t}=\mathbf{0}, \quad I=1 \ldots, N_{n}
$$

where the symbol $\boldsymbol{\Delta}$ denotes incremental quantities e.g. $\boldsymbol{\Delta} \boldsymbol{X}=\boldsymbol{X}_{(h)}-\boldsymbol{X}_{(h-1)}$, whereas the symbol $\boldsymbol{\delta}$ denotes iterative quantities e.g. $\boldsymbol{\delta} \boldsymbol{X}=\boldsymbol{X}^{(j)}-\boldsymbol{X}^{(j-1)}$. Convergence is achieved when the Euclidean norm of the equilibrium equations is sufficiently small i.e. $\left\|\boldsymbol{\delta} \boldsymbol{R}^{u(j)}\right\| \leq t_{0} l_{u}$ where $t_{o l}$ stands for the NewtonRaphson tolerance.

Finally, with the derived incremental displacement field nodal $\boldsymbol{\Delta} \mathbf{u}_{I}$, the positive part of elastic strain energy density $\psi_{e l}^{+}$is evaluated at each material point to correct the values current history field $\mathcal{H}$. Similarly, convergence is achieved when Euclidean norm of the phase field equations is small enough i.e. $\left\|\boldsymbol{R}_{(h)}^{c(k)}\right\| \leq$ tol $_{c}$ where $\operatorname{tol}_{c}$ corresponds to the phase field tolerance. This predictor-corrector procedure is continued until the convergence of both the equilibrium as well as phase field equations.

The pseudo-code is provided in Algorithm 1. In this, $E_{p}$ and $v_{p}$ correspond to the Young's modulus and Poisson's ratio respectively. Pre-existing crack paths can be defined with an initial history field $\mathcal{H}_{p_{0}}$ (see [10] for details). Furthermore, $N_{n}, N_{\text {dofs }}$ and $N_{\text {cells }}$ correspond to the total active number of grid nodes, total active unconstrained grid degree of freedom and total active cells of the Eulerian Grid respectively. These parameters, are redefined in the beginning of each time-step $h$ according to the current material point position $\mathbf{x}_{p}$. Moreover, the basis functions $\boldsymbol{N}\left(\mathbf{x}_{p_{(h)}}\right)$ and their first $\nabla \boldsymbol{N}\left(\mathbf{x}_{p_{(h)}}\right)$ and second derivatives $\Delta \boldsymbol{N}\left(\mathbf{x}_{p_{(h)}}\right)$ for all material points are evaluated in the beginning of each time-step $h$.

To evaluate the anisotropic phase field coefficient matrix (see Eq. (55)), the derivatives of basis functions are evaluated in the material principal axes. Thus, if $\left(\nabla \boldsymbol{N}\left(\mathbf{x}_{p}\right)\right)$ and $\left(\Delta \boldsymbol{N}\left(\mathbf{x}_{p}\right)\right)$ are the first and second derivatives of basis functions in global axes, then $\left(\nabla \boldsymbol{N}_{\phi_{p}}\left(\mathbf{x}_{p}\right)\right)$ and $\left(\Delta \boldsymbol{N}_{\phi_{p}}\left(\mathbf{x}_{p}\right)\right)$ are the corresponding quantities in the material principal axes of the $p^{t h}$ material point. In the two-dimensional case, coordinate transformation from the global system to the material principal system is achieved through relation (58) below

$$
\left\{\begin{array}{l}
x_{1_{\phi_{p}}} \\
x_{2_{\phi_{p}}}
\end{array}\right\}=\left[\begin{array}{cc}
\cos \left(\phi_{p}\right) & -\sin \left(\phi_{p}\right) \\
\sin \left(\phi_{p}\right) & \cos \left(\phi_{p}\right)
\end{array}\right]\left\{\begin{array}{l}
x_{1} \\
x_{2}
\end{array}\right\}=\mathbf{x}_{\phi_{p}}=\mathbf{R}_{p} \mathbf{x}
$$


and

$$
\mathbf{R}_{p}=\left[\begin{array}{cc}
\cos \left(\phi_{p}\right) & -\sin \left(\phi_{p}\right) \\
\sin \left(\phi_{p}\right) & \cos \left(\phi_{p}\right)
\end{array}\right]
$$

where $\phi_{p}$ is the material orientation of the material point (counter clockwise) and $\mathbf{x}_{\phi_{p}}$ are the coords in the material principal axes.

\section{Examples}

In this section, a series of benchmark tests are examined with the proposed scheme. For verification purposes, both the Phase Field Material Point Method (MPM-PF) as well as its Finite Element counterpoart (FEM-PF) have been implemented in an in-house Fortran code.

\subsection{Squared plate under pure tension}

In this example, the case of a square plate with a horizontal notch placed under pure tension is examined. The notch is positioned at mid-height and spans the half-length of the specimen. The geometry and boundary conditions of the problem are shown in Fig. 10a. Displacements in segment BC are restrained along $Y$ whereas a pin support is considered at point $\mathrm{O}$ restraining displacement in both directions. A load is applied at point $A$ along $Y$. All points in segment $A B$ - with the exception of $B$ - are restrained so that their corresponding displacement components along $Y$ are equal. The restrain is achieved by using a Penalty method with penalty parameter $\alpha=1000000$ [6] (see Appendix of [20] for imposing kinematical constrains with Penalty method in MPM). Plain strain conditions are assumed with the elastic isotropic properties of the material, $E=1000000 \mathrm{kN} / \mathrm{m}^{2}$ and $\nu=0.3$ for the Young modulus and Poisson's ratio respectively.

To gain insight the influence of surface energy into crack path, two cases are considered, namely that of cubic and orthotropic symmetry. The corresponding material properties are presented in Table 1. Furthermore, the polar plot of their surface energy density $\mathcal{G}_{c}(\theta)$ and their reciprocals $1 / \mathcal{G}_{c}(\theta)$ for material orientation $\phi=0$ are represented in Figs. 10b and 10c respectively. For cubic symmetry the maximum and minimum surface energy density are $\mathcal{G}_{c_{\text {max }}}=1.1575 \mathrm{kN} / \mathrm{m}$ and $\mathcal{G}_{c_{\text {min }}}=0.7071 \mathrm{kN} / \mathrm{m}$ respectively. For the case of orthotropic symmetry these are $\mathcal{G}_{c_{\max }}=1.2292 \mathrm{kN} / \mathrm{m}$ and $\mathcal{G}_{c_{\text {min }}}=0.7071$ $\mathrm{kN} / \mathrm{m}$ respectively.

A displacement control Newton Raphson scheme is utilized with the maximum value of the monitored displacement $u=0.006 \mathrm{~m}$. A force based convergence criterion is employed with a corresponding tolerance $t_{o} l_{u}=1 e-7$. A staggered solution procedure has been implemented with a single prediction step $\left(N_{\text {staggs }}=1\right)$

The problem is solved using both the FEM-PF method and the MPM-PF method. Results obtained by both solution approaches also agree with the results provided in [21] where however a purely meshless solution procedure was 
Data: Define computational grid, material point properties $\left(\mathbf{x}_{p_{(0)}}\right.$, $\left.\Omega_{p_{(0)}}, E_{p}, \nu_{p}, l_{0_{p}}, k_{p}, \overline{\mathcal{G}}_{c_{p}}, \gamma_{i j k l_{p}}, \phi_{p}, \mathcal{H}_{p_{(0)}}, \boldsymbol{\sigma}_{p_{(0)}}, \varepsilon_{p_{(0)}}\right)$

for each time step $h=1,2, . ., N_{\text {steps }}$ do

Reset the computational grid: Find active part of Eulerian Grid, $N_{n}, N_{\text {dofs }}, N_{\text {cells }}$

Compute: $\boldsymbol{N}\left(\mathbf{x}_{p_{(h)}}\right), \nabla \boldsymbol{N}\left(\mathbf{x}_{p_{(h)}}\right)$ and $\Delta \boldsymbol{N}\left(\mathbf{x}_{p_{(h)}}\right)$, for all material points. ;

Compute: $\nabla \boldsymbol{N}_{\phi_{p}}\left(\mathbf{x}_{p_{(h)}}\right)$ and $\Delta \boldsymbol{N}_{\phi_{p}}\left(\mathbf{x}_{p_{(h)}}\right)$, for all material points. ;

Define: $\boldsymbol{\delta} \boldsymbol{R}^{u(1)}=\boldsymbol{\Delta} \boldsymbol{F}_{(h)}^{\text {ext }}$;

for each staggered iteration $k=1,2, . ., N_{\text {staggs }}$ do

Compute: $\boldsymbol{F}_{(h)}^{c(k)}$ (see Eq. (56)). ;

Compute: $\boldsymbol{K}^{c}$ (see Eq. (55)). according to $\boldsymbol{N}\left(\mathbf{x}_{p_{(h)}}\right)$,

$\nabla \boldsymbol{N}_{\phi_{p}}\left(\mathbf{x}_{p_{(h)}}\right)$ and $\Delta \boldsymbol{N}_{\phi_{p}}\left(\mathbf{x}_{p_{(h)}}\right)$;

Solve: $\boldsymbol{K}^{c} \boldsymbol{c}_{(h)}^{(k)}=\boldsymbol{F}_{(h)}^{c(k)}$;

Map phase field $\left(\boldsymbol{c}_{(h)}^{(k)}\right)$ from grid nodes to material points.

Evaluate: $c_{p_{(h)}}^{(k)}, \nabla c_{p_{(h)}}^{(k)}, \Delta c_{p_{(h)}}^{(k)}, g_{p_{(h)}}^{(k)}$, for all material points (see Eq. (46), (47),(48) and (8)). ;

Initialize $\boldsymbol{\Delta} \mathbf{u}^{(0)}=\mathbf{0}$;

for each inner iteration $j=1,2, . ., N_{\text {iters }}$ do

Compute stiffness matrix of the structure: $\boldsymbol{K}^{u}$ (for constitutive matrix see Eq. (23)) ;

Solve: $\boldsymbol{K}^{u} \delta \mathbf{u}^{(j)}=\boldsymbol{\delta} \boldsymbol{R}^{u(j)}$, with displacement contol. ;

Compute: $\boldsymbol{\Delta} \mathbf{u}^{(j)}=\boldsymbol{\Delta} \mathbf{u}^{(j-1)}+\delta \mathbf{u}^{(j)}$;

Compute: $\boldsymbol{\Delta} \varepsilon_{p}^{(j)}$, for all material points (see Eq. (2)). ;

Compute: $\varepsilon_{p_{(h)}^{(j)}}=\varepsilon_{p_{(h-1)}}+\Delta \varepsilon_{p}^{(j)}$, for all material points. ;

Compute: $\boldsymbol{\sigma}_{p_{(h)}}^{(j)}$, for all material points (see Eq. (15)) ;

Compute: $\boldsymbol{\Delta} \boldsymbol{F}^{\text {int }(j)}=\left\{\boldsymbol{\Delta} \boldsymbol{F}_{I}^{\text {int }}\right\}$,

$\boldsymbol{\Delta} \boldsymbol{F}_{I}^{i n t}=\sum_{p=1}^{N_{p}} \Omega_{p}^{(j)}\left(\boldsymbol{\sigma}_{p_{(h)}}^{(j)}-\boldsymbol{\sigma}_{p_{(h-1)}}\right) \cdot \nabla N_{I}\left(\mathbf{x}_{p_{(h)}}\right) ;$

Compute Residual (Displacement-Field):

$\boldsymbol{\delta} \boldsymbol{R}^{u(j)}=\boldsymbol{\Delta} \boldsymbol{F}_{(h)}^{e x t}-\boldsymbol{\Delta} \boldsymbol{F}^{i n t(j)} ;$

Convergence Check (Displacement Field): If $\left\|\boldsymbol{\delta} \boldsymbol{R}^{u(j)}\right\| \leq t_{o} l_{u}$ or $j \geq N_{\text {iters }}$ then "exit" from loop else $j=j+1$ go to next inner iteration. ;

end

Compute: $\psi_{e l_{p_{(h)}}^{+}}^{+}$, for all material points (see Eq. (18))

$\rightarrow \mathcal{H}_{p_{(h)}}= \begin{cases}\psi_{e l_{p_{(h)}}}^{+}, & \text {for } \psi_{e l_{p_{(h)}}}^{+}>\mathcal{H}_{p_{(h-1)}} \\ \mathcal{H}_{p_{(h-1)},}, & \text { otherwise }\end{cases}$

Compute Residual (Phase-Field): $\boldsymbol{R}_{(h)}^{c(k)}$ (see Eq. (49)) according to $c_{p_{(h)}}^{(k)}, \nabla c_{p_{(h)}}^{(k)}, \Delta c_{p_{(h)}}^{(k)}, \mathcal{H}_{p}$;

Convergence Check (Phase Field): If $\left\|\boldsymbol{R}_{(h)}^{c(k)}\right\| \leq t_{c} l_{c}$ or $k \geq N_{\text {staggs }}$ then "exit" from loop else $k=k+1$ go to next stagger iteration. ;

end

Compute: $\boldsymbol{\Delta} \mathbf{u}_{p_{(h)}}=\sum_{I=1}^{N_{n}} N_{I}\left(\mathbf{x}_{p_{(h)}}\right) \boldsymbol{\Delta} \mathbf{u}_{I}^{(j)}$, for all material points. ;

Compute: $\mathbf{u}_{p_{(h)}}=\mathbf{u}_{p_{(h-1)}}+\Delta \mathbf{u}_{p_{(h)}}$, for all material points. ;

end

Compute: $\mathbf{x}_{p_{(h)}}=\mathbf{x}_{p_{(h-1)}}+\Delta \mathbf{u}_{p_{(h)}}$, for all material points. ;

Algorithm 1: Anisotropic Phase-Field Material Point Method pseudo-code (Stagger Solution Algorithm). 
adopted. The finite element mesh comprises 29929 quadratic $C^{1}$-continuous patches with a mesh size $h=0.00578 \mathrm{~m}$. A 3x3 quadrature rule is used for the quadratic basis functions as it is suggested in [18]. The MPM-PF mesh consists of 32041 back-ground quadratic $C^{1}$-continuous cells (patches) with a mesh size $h=0.00578 \mathrm{~m}$. The initial cell density utilized is $3 \times 3=9$ material points per cell. All the simulation parameters are summarized in Table 1.

Eleven different material orientation angles are considered, namely

$$
\phi=\left\{-50^{\circ},-40^{\circ},-30^{\circ},-20^{\circ},-10^{\circ}, 0^{\circ},+10^{\circ},+20^{\circ},+30^{\circ},+40^{\circ},+50^{\circ}\right\}
$$

The resulting load displacement paths for the case of $\phi=0^{\circ}$ and $\phi=-50^{\circ}$ are shown in Figs. 11a and 12a for the case of cubic and orthotropic symmetry respectively. Results between the MPM and FEM are in perfect agreement, as also shown by the corresponding relative divergence shown in Figs. 11b and 12b. In both angles, the relative divergence is smaller than $1 \%$. However, as the imposed displacement increases the divergence increases accordingly. This is attributed to the fact that in MPM-PF the position of sampling points, i.e., the material points, is updated. Conversely, the crack geometry is also updated. The variation of the ultimate limit load as a function of the material orientation angle is shown in Figs. 13a and 13b for cubic and orthotropic symmetry respectively. Results derived from the MPM implementation agree with FEM.

Figs. 14a-14d and 14e-14h represent the phase field evolution for cubic symmetry and different values of material orientation $\phi$ for both FEM-PF and MPM-PF respectively. Similarly, Figs. 15a-15d and 15e-15h represent the phase field for orthotropic symmetry of both FEM-PF and MPM-PF respectively.

The figures show that when $\phi<45^{\circ}$, the crack path evolves in the lower half of the plate for both the cases of cubic and orthotropic symmetry (see Figs. 14a-14c,14e-14g and 15a-15c,15e-15g). However, when the material orientation angle increases, i.e., when $45<\phi<90^{\circ}$, then the crack path propagates along the higher half of the plate for cubic symmetry (see Figs. 14d,14h) and in the lower half of the plate for orthotropic symmetry (see Figs. 15d,15h). This pattern is anticipated due to four-fold and two-fold symmetry aspects of cubic symmetry and orthotropic materials respectively.

\subsection{Three point layered bending test}

The case of a three point bending test on a composite beam is considered in this example. The geometry and boundary conditions of the specimen are shown in Fig. 16a. Plain strain conditions are assumed. The beam comprises two layers, namely $A$ and $B$. The two layers have similar material properties as shown in Table 2. The elastic isotropic material properties are the same as in the previous example (see section 4.1). 


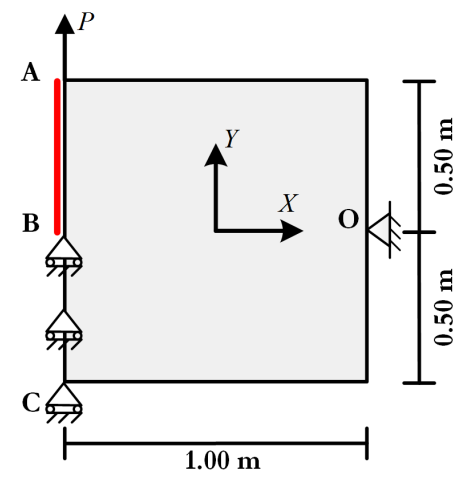

(a)

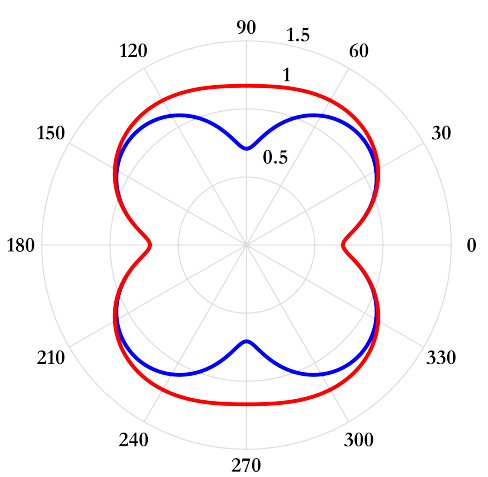

$-\mathcal{G}_{c}(\theta)$ for Cubic Symmetry

- $\mathcal{G}_{c}(\theta)$ for Orthotropic Symmetry

(b)

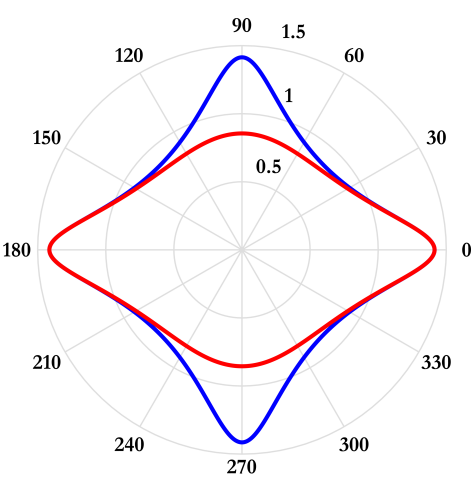

- $1 / \mathcal{G}_{c}(\theta)$ for Cubic Symmetry

$-1 / \mathcal{G}_{c}(\theta)$ for Orthotropic Symmetry

(c)

Fig. 10 Squared plate under pure tension: (a) Geometry and boundary conditions. (b) Surface energy densities and (c) their Reciprocals for material orientation $\phi=0$ in polar coordinates.

Cubic symmetry is considered for the surface energy density in both layers. The polar plots of the surface energy density and its reciprocal are shown in Figs. 16b and 16c respectively. The anisotropic parameters considered are presented in Table 2. Three different scenaria are examined for the material orientation of the two layers. In cases 1 and 2 the material orientations of both layers are $\phi=0^{\circ}$ (case 1) and $\phi=-15^{\circ}$ (case 2) respectively. In case 3 the material orientation of layer $A$ is $\phi=-15^{\circ}$ and layer $B$ is $\phi=+15^{\circ}$.

A displacement control non-linear static analysis scheme is utilized with a constant displacement increment $\Delta u \approx 0.0033 \mathrm{~mm}$ at midspan for 1550 steps. Staggered solution algorithm is utilized with a single prediction step $\left(N_{\text {staggs }}=1\right)$. A FEM-PF analysis is also made for verification purposes. The 


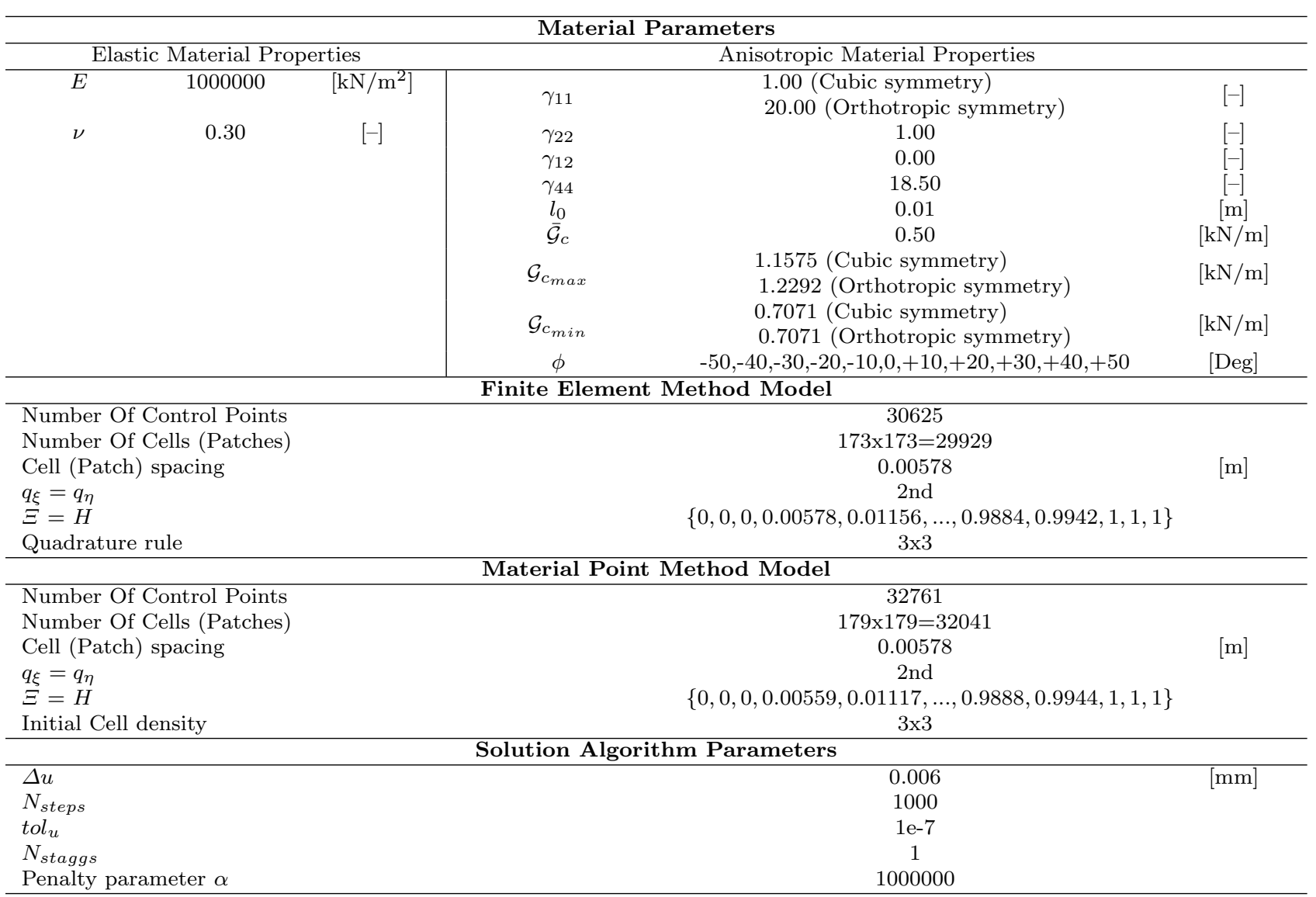

Table 1 Squared plate under pure tension: Simulation parameters.

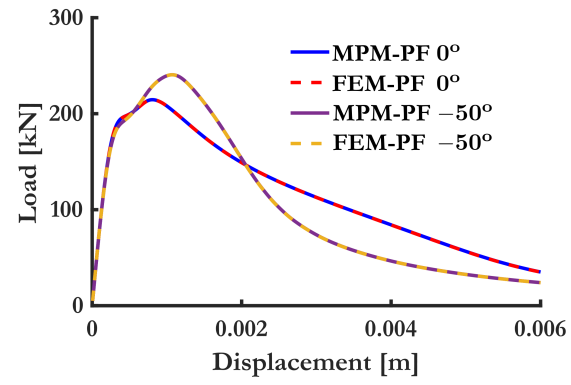

(a)

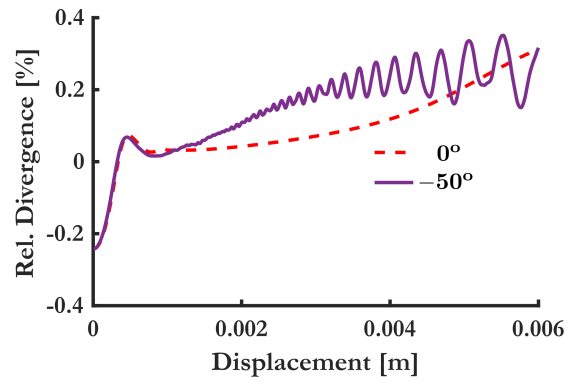

(b)

Fig. 11 Squared plate under pure tension: (a) FEM-PF vs MPM-FEM comparison and (b) relative divergence for cubic symmetry. 


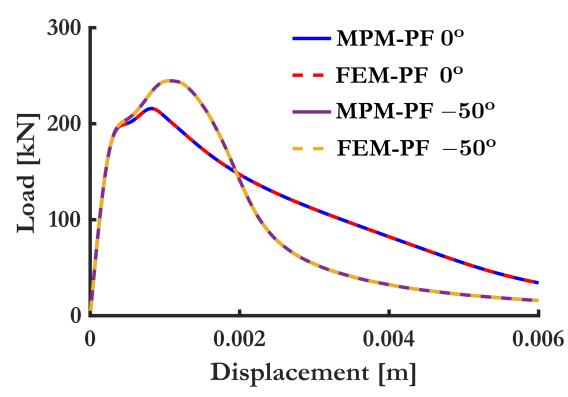

(a)

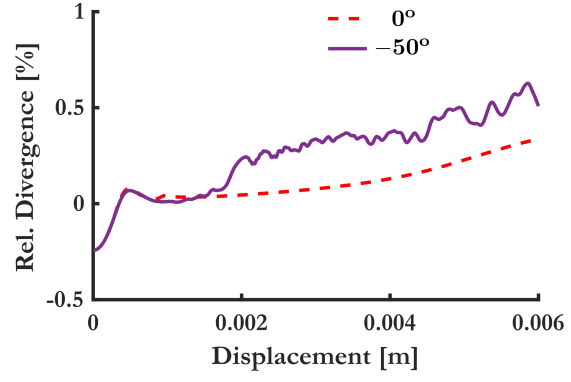

(b)

Fig. 12 Squared plate under pure tension: (a) FEM-PF vs MPM-FEM comparison and (b) relative divergence for orthotropic symmetry.

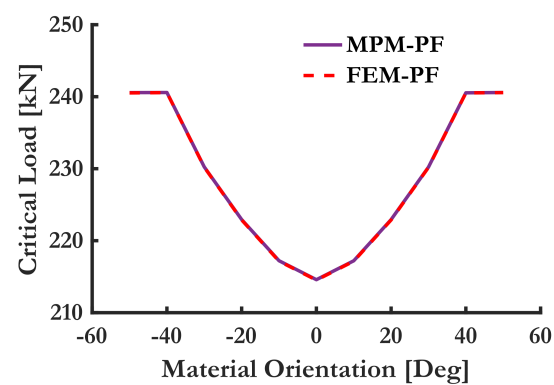

(a)

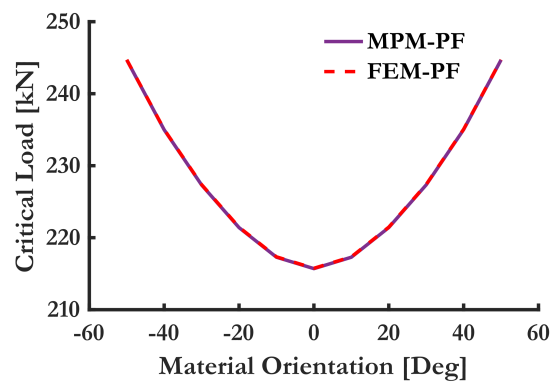

(b)

Fig. 13 Squared plate under pure tension: Critical Load vs Material Orientation for (a) cubic symmetry and (b) orthotropic symmetry.

simulation parameters of both MPM-PF and FEM-PF are summarized into Table 2.

The corresponding load paths are shown in Figs. 17. The two methods demonstrate very good agreement for all three cases. In Figs. 18a,18c and 18e the phase field distribution retrieved from FEM-PF for case 1, case 2 and case 3 respectively is shown. The corresponding results from the MPM-PF are shown in Figs. 18b,18d and 18f. All the results in Figs. 18 are retrieved at a vertical displacement $u=5 \mathrm{~mm}$.

The crack paths derived from both methods are practically identical. In case 1 - with a material orientation angle $-\phi=0^{\circ}$ - (see Figs. 18a and 18b), the crack propagates along the $Y$ axis, exactly as in the case of isotropic surface energy. However, in case 2 (see Figs. $18 \mathrm{c}$ and 18d) the anisotropic surface energy forces the crack to diverge from the $Y$ axis. Finally, in case 3 (see Figs. $18 \mathrm{e}$ and 18f) a crack 'kick' is observed in the interface of two layers as a result of their different material orientations $\phi$. The evolution of the hydrostatic stress for case 3 and MPM-PF implementation is shown in Figs. 19 for three 


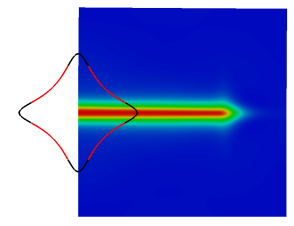

(a)

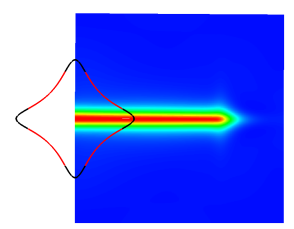

(e)

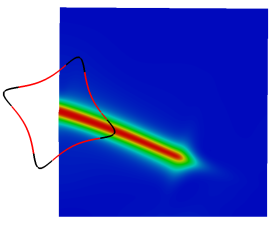

(b)

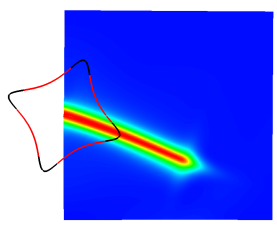

(f)

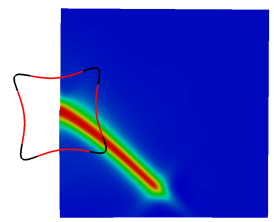

(c)

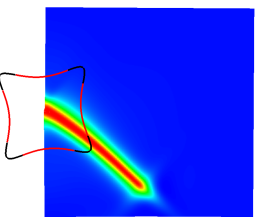

(g)

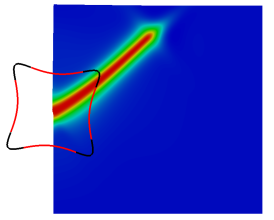

(d)

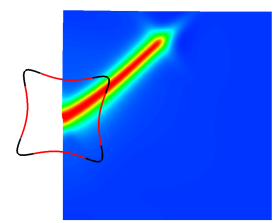

(h)

Phase Field

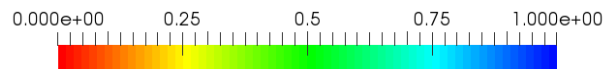

Fig. 14 Squared plate under pure tension: Phase field for $u=0.006 \mathrm{~m}$ and cubic symmetry Finite Element Method for material orientation (a) $0^{\circ}$, (b) $+20^{\circ}$, (c) $+40^{\circ}$ and (d) $+50^{\circ}$. Material Point Method for material orientation (e) $0^{\circ}$, (f) $+20^{\circ}$, (g) $+40^{\circ}$ and $(\mathrm{h})+50$.

timesteps, $u=4.6 \mathrm{~mm}, u=4.7 \mathrm{~mm}$ and $u=4.9 \mathrm{~mm}$ respectively. From these figures it can be verified that the crack propagates only due to tension as a result of the strain energy density $\psi_{e l}$ decomposition. 


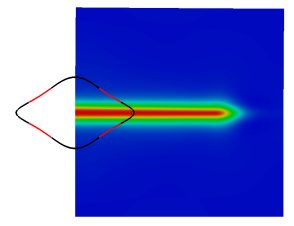

(a)

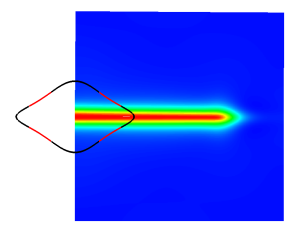

(e)

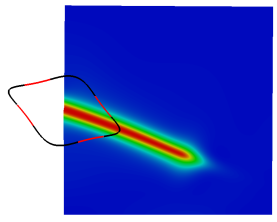

(b)

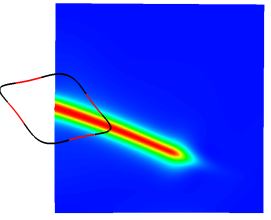

(f)

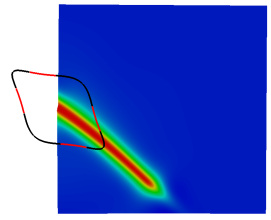

(c)

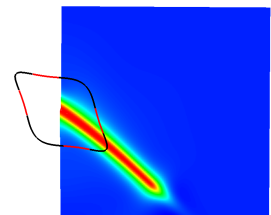

(g)

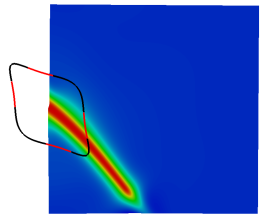

(d)

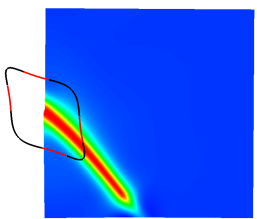

(h)

Phase Field

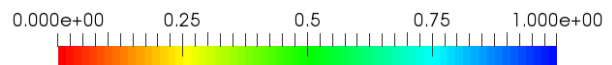

Fig. 15 Squared plate under pure tension: Phase field for $u=0.006 \mathrm{~m}$ and orthotropic symmetry. Finite Element Method for material orientation (a) $0^{\circ}$, (b) $+20^{\circ}$, (c) $+40^{\circ}$ and (d) $+50^{\circ}$. Material Point Method for material orientation (e) $0^{\circ}$, (f) $+20^{\circ}$, (g) $+40^{\circ}$ and (h) +50 . 


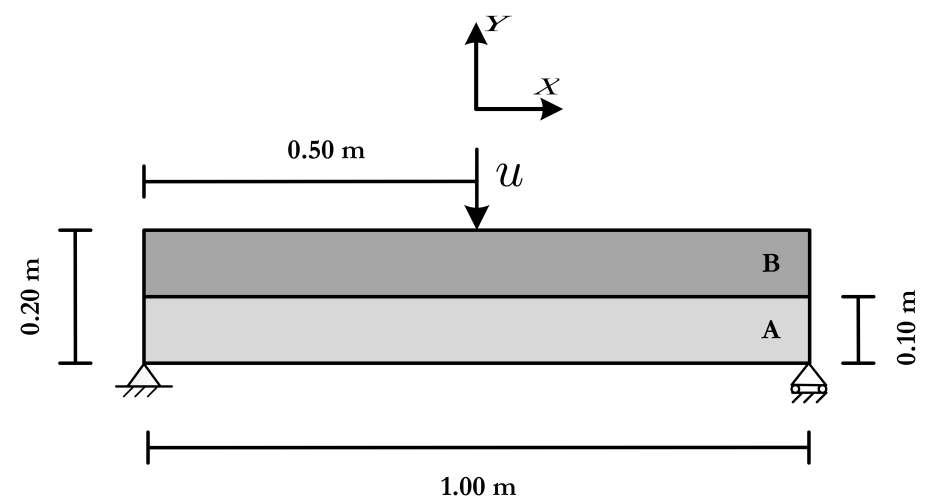

(a)

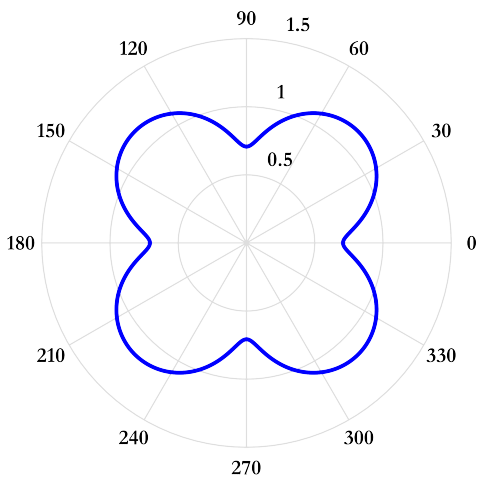

- $\mathcal{G}_{c}(\theta)$ for Cubic Symmetry

(b)

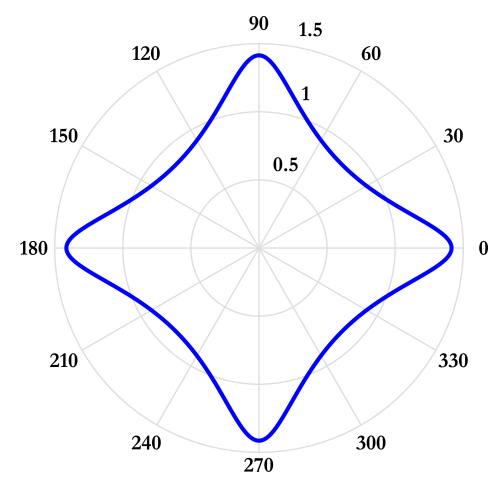

- $1 / \mathcal{G}_{c}(\theta)$ for Cubic Symmetry

(c)

Fig. 16 Three point layered bending test: (a) Geometry and boundary conditions. (b) Surface energy density and (c) its Reciprocal for material orientation $\phi=0$ in polar coordinates. 
Material Parameters

\begin{tabular}{|c|c|c|c|c|c|c|c|c|}
\hline \multicolumn{9}{|c|}{ Material Parameters } \\
\hline \multicolumn{4}{|c|}{ Elastic Material Properties } & \multicolumn{5}{|c|}{ Anisotropic Material Properties } \\
\hline & Layer A & Layer B & & & & Layer A & Layer B & \\
\hline$E$ & $\overline{1000000}$ & $\overline{1000000}$ & {$\left[\mathrm{kN} / \mathrm{m}^{2}\right]$} & $\gamma_{11}$ & & 1.00 & 1.00 & {$[-]$} \\
\hline \multirow[t]{10}{*}{$\nu$} & 0.30 & 0.30 & {$[-]$} & $\gamma_{22}$ & & 1.00 & 1.00 & {$[-]$} \\
\hline & & & & $\gamma_{12}$ & & 0.00 & 0.00 & {$[-]$} \\
\hline & & & & $\gamma_{44}$ & & 18.50 & 18.50 & {$[-]$} \\
\hline & & & & $l_{0}$ & & 0.005 & 0.005 & {$[\mathrm{~m}]$} \\
\hline & & & & $\overline{\mathcal{G}}_{c}$ & & 0.50 & 0.50 & {$[\mathrm{kN} / \mathrm{m}]$} \\
\hline & & & & $\mathcal{G}_{c_{\max }}$ & & 1.1575 & 1.1575 & {$[\mathrm{kN} / \mathrm{m}]$} \\
\hline & & & & $\mathcal{G}_{c_{\min }}$ & & 0.7071 & 0.7071 & {$[\mathrm{kN} / \mathrm{m}]$} \\
\hline & & & & & Case 1 & 0 & 0 & \\
\hline & & & & $\phi$ & Case 2 & -15 & -15 & [Deg] \\
\hline & & & & & Case 3 & -15 & +15 & \\
\hline \multicolumn{9}{|c|}{ Finite Element Method Model } \\
\hline \multicolumn{4}{|c|}{ Number Of Control Points } & \multicolumn{5}{|c|}{41584} \\
\hline \multicolumn{4}{|c|}{ Number Of Cells (Patches) } & \multicolumn{5}{|c|}{$450 \times 90=40500$} \\
\hline \multicolumn{4}{|c|}{ Cell (Patch) spacing } & \multicolumn{4}{|c|}{0.0022} & {$[\mathrm{~m}]$} \\
\hline \multicolumn{4}{|c|}{$q_{\xi}=q_{\eta}$} & \multicolumn{4}{|c|}{ 2nd } & \\
\hline \multicolumn{4}{|c|}{$\stackrel{4 \xi}{\Xi}-9 \eta$} & \multicolumn{5}{|c|}{$0,0,0,0.0022,0.0044, \ldots, 0.9955,0.9977,1,1,1$} \\
\hline \multicolumn{4}{|c|}{$H$} & \multicolumn{5}{|c|}{$0,0,0,0.0111,0.0222, \ldots, 0.9777,0.9888,1,1,1$} \\
\hline & \multicolumn{5}{|c|}{$3 \times 3$} \\
\hline \multicolumn{4}{|c|}{ Quadrature rule } & \multicolumn{5}{|c|}{ Material Point Method Model } \\
\hline \multicolumn{4}{|c|}{ Number Of Control Points } & \multicolumn{5}{|c|}{44884} \\
\hline \multicolumn{4}{|c|}{ Number Of Cells (Patches) } & \multicolumn{5}{|c|}{$456 \times 96=43776$} \\
\hline \multicolumn{4}{|c|}{ Cell (Patch) spacing } & \multicolumn{4}{|c|}{0.0022} & {$[\mathrm{~m}]$} \\
\hline$q_{\xi}=q$ & & & & & & & & \\
\hline$\Xi$ & & & & & & $21,0.0043$ & $78,1,1,1\}$ & \\
\hline$H$ & & & & & & $04,0.0208$ & $95,1,1,1\}$ & \\
\hline Initial & density & & & & & & & \\
\hline & & & & Solutic & lgorith & neters & & \\
\hline$\Delta u$ & & & & & & & & {$[\mathrm{~mm}]$} \\
\hline$N_{\text {step }}$ & & & & & & & & \\
\hline $\operatorname{tol}_{u}$ & & & & & & & & \\
\hline$N_{\text {stag }}$ & & & & & & & & \\
\hline
\end{tabular}

Table 2 Three point layered bending test: Simulation parameters. 


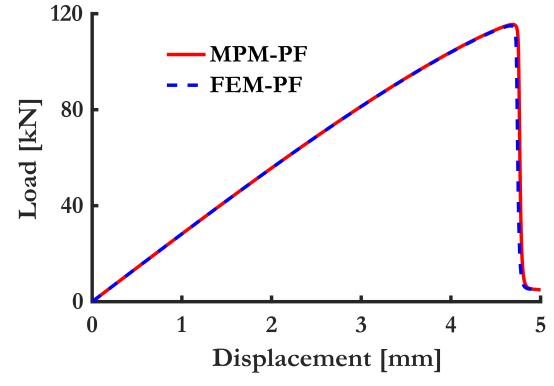

(a)

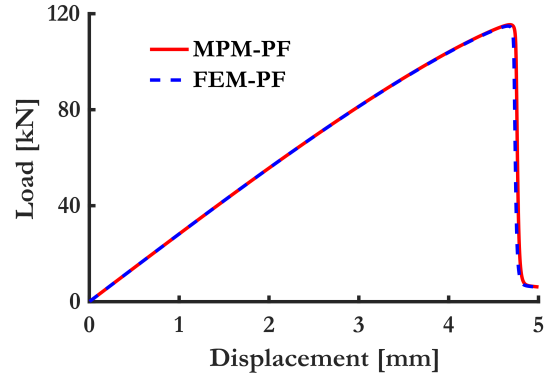

(b)

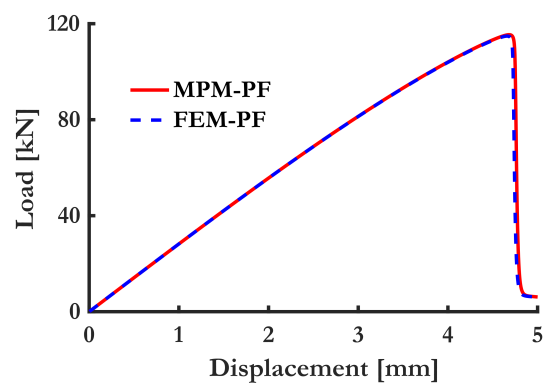

(c)

Fig. 17 Three point layered bending test: Load displacement paths for (a) Case 1 (b) Case 2 (c) Case 3 


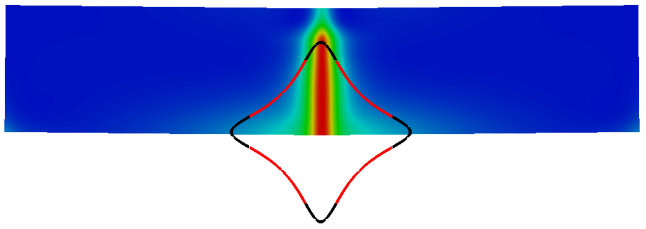

(a)

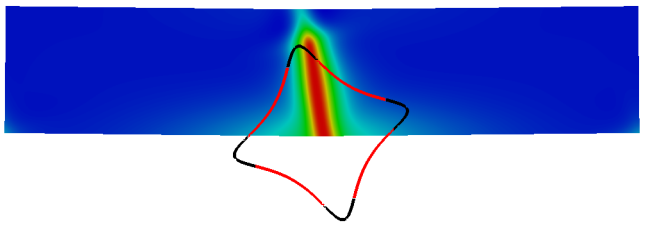

(c)

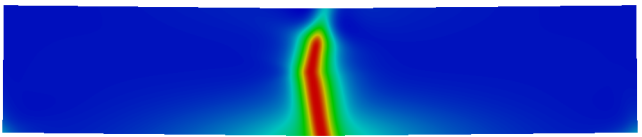

(e)

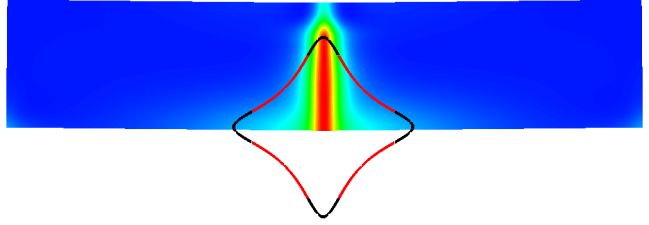

(b)

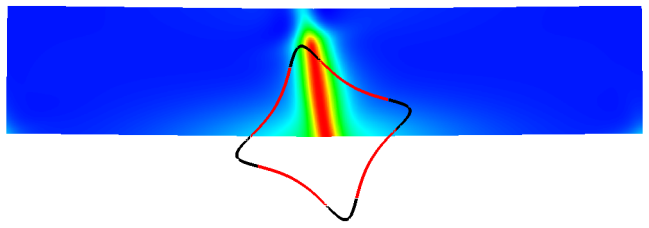

(d)

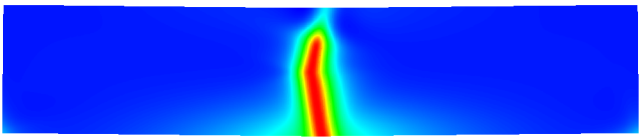

(f)

Phase Field

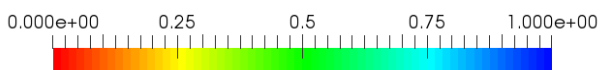

Fig. 18 Three point layered bending test: Phase field for $u=5 \mathrm{~mm}$. Finite Element Method for (a) case 1, (c) case 2 and (e) case 3 respectively. Material Point Method for (b) case 1 , (d) case 2 and (f) case 3 respectively. 


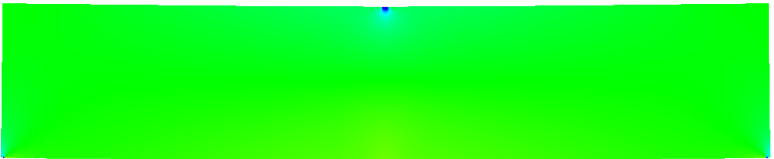

(a)

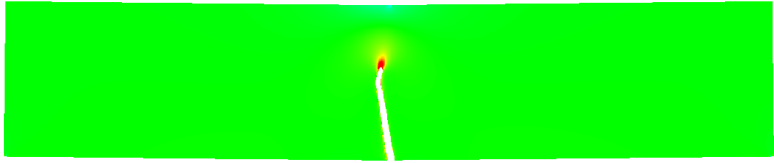

(b)

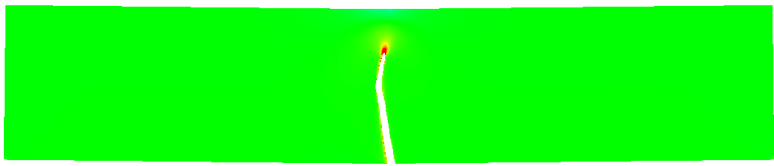

(c)

Hydrostatic Stress (kPa)

$-1.000 e+04-5000 \quad 0 \quad 5000 \quad 1.000 e+04$

Fig. 19 Three point layered bending test: Hydrostatic Stress (case 3) for (a) $u=4.6 \mathrm{~mm}$, (b) $u=4.7 \mathrm{~mm}$ and (c) $u=5.0 \mathrm{~mm}$ respectively. Material Points with $c_{p}<0.02$ have been removed. 


\section{Conclusions}

A robust framework for the resolution of evolving fractures in brittle anisotropic materials is introduced. The method employed is based on a phase field approximation of the fracture surface energy defined on the basis of a fourth order anisotropic functional. The phase field is subsequently coupled to the material stress field by introducing the former as a degradation parameter on the elastic energy density of the material. Through a purely variation framework, the coupled partial differential equations of the problem are introduced. A Material Point Method approach is used to derive the discrete governing coupled equations of the problem. The latter are then solved in a staggered fashion. In this work, the specific cases of both cubic symmetry and orthotropy are examined in representative examples. Based on these, the proposed methodology is verified by means of comparison against the standard finite element phase field implementation. Results demonstrate that the Material Point Method performs extremely well in resolving fracture propagation problems. This work will thus form the basis for subsequent derivations pertaining to large displacement dynamic problems where Material Point Methods have proven advantageous as compared to finite element methods.

\section{Appendix A Variational approach of the anisotropic phase-field model}

In the energy balance equation (9) the rate of the kinetic energy is evaluated as

$$
\dot{\mathcal{K}}(\dot{\mathbf{u}})=\frac{d}{d t} \int_{\Omega} \frac{1}{2} \rho|\dot{\mathbf{u}}|^{2} d \Omega=\int_{\Omega}([\rho \ddot{\mathbf{u}}] \cdot \dot{\mathbf{u}}) d \Omega
$$

Similarly, the rate of the external work is expressed as

$$
\dot{\mathcal{W}}^{e x t}(\dot{\mathbf{u}})=\int_{\partial \Omega_{\bar{t}}}(\overline{\boldsymbol{t}} \cdot \dot{\mathbf{u}}) d \partial \Omega_{\bar{t}}+\int_{\Omega}(\boldsymbol{b} \cdot \dot{\mathbf{u}}) d \Omega
$$

and the rate of the internal work is defined accordingly as

$$
\dot{\mathcal{W}}^{\text {int }}(\dot{\mathbf{u}}, \dot{c}, \nabla \dot{c})=\frac{d \Psi_{p o t}}{d t}=\frac{d}{d t} \int_{\Omega}\left(\psi_{e l}+\overline{\mathcal{G}}_{c} \mathcal{Z}_{c, A n i s}\right) d \Omega
$$

Applying the divergence theorem in equation (63), the rate of the internal work $\dot{\mathcal{W}}^{\text {int }}(\dot{\mathbf{u}}, \dot{c}, \nabla \dot{c})$ assumes the following form

$$
\dot{\mathcal{W}}^{\text {int }}(\dot{\mathbf{u}}, \dot{c}, \nabla \dot{c})=\mathcal{B}_{1}+\mathcal{B}_{2}+\mathcal{B}_{3}+\mathcal{B}_{4}
$$

where the components $B_{i}, i=1 \ldots 4$ assume the following expressions

$$
\begin{aligned}
\mathcal{B}_{1} & =\frac{d}{d t} \int_{\Omega} \psi_{e l} d \Omega \\
& =\int_{\partial \Omega}([\boldsymbol{\sigma} \boldsymbol{n}] \cdot \dot{\mathbf{u}}) d \partial \Omega-\int_{\Omega}([\nabla \cdot \boldsymbol{\sigma}] \cdot \dot{\mathbf{u}}) d \Omega+\int_{\Omega}\left(\psi_{e l_{c}} \dot{c}\right) d \Omega
\end{aligned}
$$




$$
\begin{aligned}
\mathcal{B}_{2}= & \frac{d}{d t} \int_{\Omega}\left(\overline{\mathcal{G}}_{c}\left[\frac{(c-1)^{2}}{4 l_{0}}\right]\right) d \Omega=\int_{\Omega}\left(\left[\overline{\mathcal{G}}_{c} \frac{(c-1)}{2 l_{0}}\right] \dot{c}\right) d \Omega \\
\mathcal{B}_{3} & =\frac{d}{d t} \int_{\Omega}\left(\overline{\mathcal{G}}_{c}\left[l_{0}|\nabla c|^{2}\right]\right) d \Omega \\
& =\int_{\partial \Omega}\left(\left[\overline{\mathcal{G}}_{c} l_{0} 2 \nabla c\right] \cdot \boldsymbol{n} \dot{c}\right) d \partial \Omega-\int_{\Omega}\left(\left[\overline{\mathcal{G}}_{c} l_{0} 2 \Delta c\right] \dot{c}\right) d \Omega
\end{aligned}
$$

and

$$
\begin{aligned}
\mathcal{B}_{4} & =\frac{d}{d t} \int_{\Omega}\left(\overline{\mathcal{G}}_{c}\left[l_{0}^{3} \sum_{i j k l} \gamma_{i j k l} \frac{\partial^{2} c}{\partial x_{i} \partial x_{j}} \frac{\partial^{2} c}{\partial x_{i} \partial x_{j}}\right]\right) d \Omega \\
& =\int_{\Omega}\left(\overline{\mathcal{G}}_{c}\left[l_{0}^{3} \sum_{i j k l} \gamma_{i j k l} \frac{d}{d t}\left(\frac{\partial^{2} c}{\partial x_{i} \partial x_{j}} \frac{\partial^{2} c}{\partial x_{k} \partial x_{l}}\right)\right]\right) d \Omega \\
& =\int_{\Omega}\left(\overline{\mathcal{G}}_{c}\left[l_{0}^{3} \sum_{i j k l} \gamma_{i j k l}\left(\frac{d}{d t}\left(\frac{\partial^{2} c}{\partial x_{i} \partial x_{j}}\right) \frac{\partial^{2} c}{\partial x_{k} \partial x_{l}}+\frac{\partial^{2} c}{\partial x_{i} \partial x_{j}} \frac{d}{d t}\left(\frac{\partial^{2} c}{\partial x_{k} \partial x_{l}}\right)\right)\right]\right) d \Omega \\
& =\mathcal{T}_{1}+\mathcal{T}_{2}
\end{aligned}
$$

respectively, where

$$
\mathcal{T}_{1}=\int_{\Omega}\left(\overline{\mathcal{G}}_{c}\left[l_{0}^{3} \sum_{i j k l} \gamma_{i j k l}\left(\frac{d}{d t}\left(\frac{\partial^{2} c}{\partial x_{i} \partial x_{j}}\right) \frac{\partial^{2} c}{\partial x_{k} \partial x_{l}}\right)\right]\right) d \Omega
$$

and

$$
\mathcal{T}_{2}=\int_{\Omega}\left(\overline{\mathcal{G}}_{c}\left[l_{0}^{3} \sum_{i j k l} \gamma_{i j k l}\left(\frac{\partial^{2} c}{\partial x_{i} \partial x_{j}} \frac{d}{d t}\left(\frac{\partial^{2} c}{\partial x_{k} \partial x_{l}}\right)\right)\right]\right) d \Omega
$$


Components $\mathcal{T}_{1}$ and $\mathcal{T}_{2}$ are further expanded employing the divergence theorem into

$$
\begin{aligned}
& \mathcal{T}_{1}=\int_{\partial \Omega}\left(\left[\overline{\mathcal{G}}_{c} l_{0}^{3} \sum_{i j k l} \gamma_{i j k l}\left(\frac{d}{d t}\left(\frac{\partial c}{\partial x_{i}}\right) \frac{\partial^{2} c}{\partial x_{k} \partial x_{l}}\right)\right] \cdot \boldsymbol{n}\right) d \partial \Omega-\int_{\Omega}\left(\left[\overline{\mathcal{G}}_{c} l_{0}^{3} \sum_{i j k l} \gamma_{i j k l}\left(\frac{d}{d t}\left(\frac{\partial c}{\partial x_{i}}\right) \frac{\partial^{3} c}{\partial x_{j} \partial x_{k} \partial x_{l}}\right)\right]\right) d \Omega \\
& =\int_{\partial \Omega}\left(\left[\overline{\mathcal{G}}_{c} l_{0}^{3} \sum_{i j k l} \gamma_{i j k l}\left(\frac{d}{d t}\left(\frac{\partial c}{\partial x_{i}}\right) \frac{\partial^{2} c}{\partial x_{k} \partial x_{l}}\right)\right] \cdot \boldsymbol{n}\right) d \partial \Omega \\
& -\left(\int_{\partial \Omega}\left(\left[\overline{\mathcal{G}}_{c} l_{0}^{3} \sum_{i j k l} \gamma_{i j k l}\left(\frac{d c}{d t} \frac{\partial^{3} c}{\partial x_{j} \partial x_{k} \partial x_{l}}\right)\right] \cdot \boldsymbol{n}\right) d \partial \Omega-\int_{\Omega}\left(\left[\overline{\mathcal{G}}_{c} l_{0}^{3} \sum_{i j k l} \gamma_{i j k l}\left(\frac{d c}{d t} \frac{\partial^{4} c}{\partial x_{i} \partial x_{j} \partial x_{k} \partial x_{l}}\right)\right]\right) d \Omega\right) \\
& =\int_{\partial \Omega}\left(\left[\overline{\mathcal{G}}_{c} l_{0}^{3} \sum_{i j k l} \gamma_{i j k l}\left(\frac{d}{d t}\left(\frac{\partial c}{\partial x_{i}}\right) \frac{\partial^{2} c}{\partial x_{k} \partial x_{l}}\right)\right] \cdot \boldsymbol{n}\right) d \partial \Omega \\
& -\left(\int_{\partial \Omega}\left(\left[\overline{\mathcal{G}}_{c} l_{0}^{3} \sum_{i j k l} \gamma_{i j k l}\left(\frac{\partial^{3} c}{\partial x_{j} \partial x_{k} \partial x_{l}}\right)\right] \cdot \boldsymbol{n} \dot{c}\right) d \partial \Omega-\int_{\Omega}\left(\left[\overline{\mathcal{G}}_{c} l_{0}^{3} \sum_{i j k l} \gamma_{i j k l}\left(\frac{\partial^{4} c}{\partial x_{i} \partial x_{j} \partial x_{k} \partial x_{l}}\right)\right] \dot{c}\right) d \Omega\right)
\end{aligned}
$$

and

$$
\begin{aligned}
& \mathcal{T}_{2}=\int_{\partial \Omega}\left(\left[\overline{\mathcal{G}}_{c} l_{0}^{3} \sum_{i j k l} \gamma_{i j k l}\left(\frac{\partial^{2} c}{\partial x_{i} \partial x_{j}} \frac{d}{d t}\left(\frac{\partial c}{\partial x_{l}}\right)\right)\right] \cdot \boldsymbol{n}\right) d \partial \Omega-\int_{\Omega}\left(\left[\overline{\mathcal{G}}_{c} l_{0}^{3} \sum_{i j k l} \gamma_{i j k l}\left(\frac{\partial^{3} c}{\partial x_{i} \partial x_{j} \partial x_{k}} \frac{d}{d t}\left(\frac{\partial c}{\partial x_{l}}\right)\right)\right]\right) d \Omega \\
& =\int_{\partial \Omega}\left(\left[\overline{\mathcal{G}}_{c} l_{0}^{3} \sum_{i j k l} \gamma_{i j k l}\left(\frac{\partial^{2} c}{\partial x_{i} \partial x_{j}} \frac{d}{d t}\left(\frac{\partial c}{\partial x_{l}}\right)\right)\right] \cdot \boldsymbol{n}\right) d \partial \Omega \\
& -\left(\int_{\partial \Omega}\left(\left[\overline{\mathcal{G}}_{c} l_{0}^{3} \sum_{i j k l} \gamma_{i j k l}\left(\frac{\partial^{3} c}{\partial x_{i} \partial x_{j} \partial x_{k}} \frac{d c}{d t}\right)\right] \cdot \boldsymbol{n}\right) d \partial \Omega-\int_{\Omega}\left(\left[\overline{\mathcal{G}}_{c} l_{0}^{3} \sum_{i j k l} \gamma_{i j k l}\left(\frac{\partial^{4} c}{\partial x_{i} \partial x_{j} \partial x_{k} \partial x_{l}} \frac{d c}{d t}\right)\right]\right) d \Omega\right) \\
& =\int_{\partial \Omega}\left(\left[\overline{\mathcal{G}}_{c} l_{0}^{3} \sum_{i j k l} \gamma_{i j k l}\left(\frac{\partial^{2} c}{\partial x_{i} \partial x_{j}} \frac{d}{d t}\left(\frac{\partial c}{\partial x_{l}}\right)\right)\right] \cdot \boldsymbol{n}\right) d \partial \Omega \\
& -\left(\int_{\partial \Omega}\left(\left[\overline{\mathcal{G}}_{c} l_{0}^{3} \sum_{i j k l} \gamma_{i j k l}\left(\frac{\partial^{3} c}{\partial x_{i} \partial x_{j} \partial x_{k}}\right)\right] \cdot \boldsymbol{n} \dot{c}\right) d \partial \Omega-\int_{\Omega}\left(\left[\overline{\mathcal{G}}_{c} l_{0}^{3} \sum_{i j k l} \gamma_{i j k l}\left(\frac{\partial^{4} c}{\partial x_{i} \partial x_{j} \partial x_{k} \partial x_{l}}\right)\right] \dot{c}\right) d \Omega\right)
\end{aligned}
$$

respectively. 
Substituting equations (71) and (72) in Eq. (68) the following expression is derived for $\mathcal{B}_{4}$

$$
\begin{aligned}
\mathcal{B}_{4} & =\int_{\partial \Omega}\left(\left[\overline{\mathcal{G}}_{c} l_{0}^{3} \sum_{i j k l} \gamma_{i j k l}\left(\frac{d}{d t}\left(\frac{\partial c}{\partial x_{i}}\right) \frac{\partial^{2} c}{\partial x_{k} \partial x_{l}}\right)+\overline{\mathcal{G}}_{c} l_{0}^{3} \sum_{i j k l} \gamma_{i j k l}\left(\frac{\partial^{2} c}{\partial x_{i} \partial x_{j}} \frac{d}{d t}\left(\frac{\partial c}{\partial x_{l}}\right)\right)\right] \cdot \boldsymbol{n}\right) d \partial \Omega \\
& -\int_{\partial \Omega}\left(\left[\overline{\mathcal{G}}_{c} l_{0}^{3} \sum_{i j k l} \gamma_{i j k l}\left(\frac{\partial^{3} c}{\partial x_{j} \partial x_{k} \partial x_{l}}\right)+\overline{\mathcal{G}}_{c} l_{0}^{3} \sum_{i j k l} \gamma_{i j k l}\left(\frac{\partial^{3} c}{\partial x_{i} \partial x_{j} \partial x_{k}}\right)\right] \cdot \boldsymbol{n} \dot{c}\right) d \partial \Omega \\
& +2 \int_{\Omega}\left(\left[\overline{\mathcal{G}}_{c} l_{0}^{3} \sum_{i j k l} \gamma_{i j k l}\left(\frac{\partial^{4} c}{\partial x_{i} \partial x_{j} \partial x_{k} \partial x_{l}}\right)\right] \dot{c}\right) d \Omega
\end{aligned}
$$

Substituting Eq. (73) in the energy balance equation (9) expression (14) is finally established.

\section{Appendix B Transformation of surface energy density to polar coordinates}

The surface energy density and their corresponding reciprocal expression polar plots are evaluated according to the methodology introduced in [21]. In this, the Cartesian coordinate system $\mathbf{x}\left(x_{1}, x_{2}\right)$ is transformed to $\mathbf{x}_{\theta}\left(x_{1_{\theta}}, x_{2_{\theta}}\right)$ where the $x_{1_{\theta}}$-axis is defined along the crack path $\Gamma$ and $x_{2_{\theta}}$-axis is the axis normal to the crack interface as shown in Fig. 20. Angle $\theta$ is the counterclockwise angle between $x_{1}$-axis and $x_{1_{\theta}}$.

Thus, coordinate transformation from $\mathbf{x}\left(x_{1}, x_{2}\right)$ to $\mathbf{x}_{\theta}\left(x_{1_{\theta}}, x_{2_{\theta}}\right)$ is performed through the transformation equation (74)

$$
\left\{\begin{array}{l}
x_{1_{\theta}} \\
x_{2_{\theta}}
\end{array}\right\}=\left[\begin{array}{cc}
\cos (\theta) & -\sin (\theta) \\
\sin (\theta) & \cos (\theta)
\end{array}\right]\left\{\begin{array}{l}
x_{1} \\
x_{2}
\end{array}\right\}=\mathbf{x}_{\theta}=\mathbf{R}_{\theta} \mathbf{x}
$$

with the inverse transformation defined as

$$
\left\{\begin{array}{l}
x_{1} \\
x_{2}
\end{array}\right\}=\left[\begin{array}{cc}
\cos (\theta) & \sin (\theta) \\
-\sin (\theta) & \cos (\theta)
\end{array}\right]\left\{\begin{array}{l}
x_{1_{\theta}} \\
x_{2_{\theta}}
\end{array}\right\}=\mathbf{x}=\mathbf{R}_{\theta}^{T} \mathbf{x}_{\theta}
$$

Assuming that $c\left(\mathbf{x}\left(\mathbf{x}_{\theta}\right)\right) \approx c\left(\mathbf{x}\left(x_{2_{\theta}}\right)\right)$ and applying the chain rule, the phase field first spatial derivatives are expressed as

$$
\frac{\partial c}{\partial x_{1}}=\frac{\partial c}{\partial x_{1_{\theta}}} \frac{\partial x_{1_{\theta}}}{\partial x_{1}}+\frac{\partial c}{\partial x_{2_{\theta}}} \frac{\partial x_{2_{\theta}}}{\partial x_{1}} \approx \frac{\partial c}{\partial x_{2_{\theta}}} \frac{\partial x_{2_{\theta}}}{\partial x_{1}}=\frac{\partial c}{\partial x_{2_{\theta}}} \sin (\theta)
$$

and

$$
\frac{\partial c}{\partial x_{2}}=\frac{\partial c}{\partial x_{1_{\theta}}} \frac{\partial x_{1_{\theta}}}{\partial x_{2}}+\frac{\partial c}{\partial x_{2_{\theta}}} \frac{\partial x_{2_{\theta}}}{\partial x_{2}} \approx \frac{\partial c}{\partial x_{2_{\theta}}} \frac{\partial x_{2_{\theta}}}{\partial x_{2}}=\frac{\partial c}{\partial x_{2_{\theta}}} \cos (\theta)
$$


respectively. Similarly, the second spatial derivatives are expressed as

$$
\begin{array}{r}
\frac{\partial^{2} c}{\partial x_{1}^{2}}=\frac{\partial}{\partial x_{1}}\left(\frac{\partial c}{\partial x_{1}}\right)=\frac{\partial}{\partial x_{1_{\theta}}}\left(\frac{\partial c}{\partial x_{1}}\right) \frac{\partial x_{1_{\theta}}}{\partial x_{1}}+\frac{\partial}{\partial x_{2_{\theta}}}\left(\frac{\partial c}{\partial x_{1}}\right) \frac{\partial x_{2_{\theta}}}{\partial x_{1}} \approx \\
\frac{\partial}{\partial x_{2_{\theta}}}\left(\frac{\partial c}{\partial x_{1}}\right) \frac{\partial x_{2_{\theta}}}{\partial x_{1}}=\frac{\partial^{2} c}{\partial x_{2_{\theta}}^{2}} \sin ^{2}(\theta) \\
\frac{\partial^{2} c}{\partial x_{2}^{2}}=\frac{\partial}{\partial x_{2}}\left(\frac{\partial c}{\partial x_{2}}\right)=\frac{\partial}{\partial x_{1_{\theta}}}\left(\frac{\partial c}{\partial x_{2}}\right) \frac{\partial x_{1_{\theta}}}{\partial x_{2}}+\frac{\partial}{\partial x_{2_{\theta}}}\left(\frac{\partial c}{\partial x_{2}}\right) \frac{\partial x_{2_{\theta}}}{\partial x_{2}} \approx \\
\frac{\partial}{\partial x_{2_{\theta}}}\left(\frac{\partial c}{\partial x_{2}}\right) \frac{\partial x_{2_{\theta}}}{\partial x_{2}}=\frac{\partial^{2} c}{\partial x_{2_{\theta}}^{2}} \cos ^{2}(\theta)
\end{array}
$$

and

$$
\begin{array}{r}
\frac{\partial^{2} c}{\partial x_{1} \partial x_{2}}=\frac{\partial}{\partial x_{1}}\left(\frac{\partial c}{\partial x_{2}}\right)=\frac{\partial}{\partial x_{1_{\theta}}}\left(\frac{\partial c}{\partial x_{2}}\right) \frac{\partial x_{1_{\theta}}}{\partial x_{1}}+\frac{\partial}{\partial x_{2_{\theta}}}\left(\frac{\partial c}{\partial x_{2}}\right) \frac{\partial x_{2_{\theta}}}{\partial x_{1}} \approx \\
\frac{\partial}{\partial x_{2_{\theta}}}\left(\frac{\partial c}{\partial x_{2}}\right) \frac{\partial x_{2_{\theta}}}{\partial x_{1}}=\frac{\partial^{2} c}{\partial x_{2_{\theta}}^{2}} \cos (\theta) \sin (\theta) \approx \frac{\partial^{2} c}{\partial x_{2} \partial x_{1}}
\end{array}
$$

respectively. Higher order spatial derivatives are defined accordingly as

$$
\frac{\partial^{3} c}{\partial x_{1}^{3}} \approx \frac{\partial^{3} c}{\partial x_{2_{\theta}}^{3}} \sin ^{3}(\theta) \quad \text { and } \quad \frac{\partial^{3} c}{\partial x_{2}{ }^{3}} \approx \frac{\partial^{3} c}{\partial x_{2_{\theta}}^{3}} \cos ^{3}(\theta)
$$

and

$$
\begin{array}{rr}
\frac{\partial^{4} c}{\partial x_{1}{ }^{4}} \approx \frac{\partial^{4} c}{\partial x_{2_{\theta}}^{4}} \sin ^{4}(\theta), \quad \frac{\partial^{4} c}{\partial x_{2}{ }^{4}} \approx \frac{\partial^{4} c}{\partial x_{2_{\theta}}^{4}} \cos ^{4}(\theta) \quad, \quad \frac{\partial^{4} c}{\partial x_{1}^{2} \partial x_{2}^{2}} \approx \frac{\partial^{4} c}{\partial x_{2_{\theta}}^{4}} \cos ^{2}(\theta) \sin ^{2}(\theta) \\
\frac{\partial^{4} c}{\partial x_{1} \partial x_{2}{ }^{3}} \approx \frac{\partial^{4} c}{\partial x_{2_{\theta}}^{4}} \cos ^{3}(\theta) \sin (\theta) & , \quad \frac{\partial^{4} c}{\partial x_{2} \partial x_{1}{ }^{3}} \approx \frac{\partial^{4} c}{\partial x_{2_{\theta}}^{4}} \sin ^{3}(\theta) \cos (\theta)
\end{array}
$$

Employing equations (76) to (82), the functional $\mathcal{Z}_{c, \text { Anis }}$ of equation (4) is expressed in polar coordinates as

$$
\begin{aligned}
& \mathcal{Z}_{c, \text { Anis }}=\left[\frac{(c-1)^{2}}{4 l_{0}}+l_{0}|\nabla c|^{2}+l_{0}^{3} \sum_{i j k l} \gamma_{i j k l} \frac{\partial^{2} c}{\partial x_{i} \partial x_{j}} \frac{\partial^{2} c}{\partial x_{k} \partial x_{l}}\right] \approx \\
& {\left[\frac{(c-1)^{2}}{4 l_{0}}+l_{0}\left(\frac{\partial c}{\partial x_{2_{\theta}}}\right)^{2}+l_{0}^{3} \gamma_{\theta}\left(\frac{\partial^{2} c}{\partial x_{2_{\theta}}^{2}}\right)^{2}\right]}
\end{aligned}
$$

where

$$
\begin{array}{r}
\gamma_{\theta}=\gamma_{1111} \sin ^{4}(\theta)+\gamma_{2222} \cos ^{4}(\theta)+\gamma_{1212} \cos ^{2}(\theta) \sin ^{2}(\theta)+ \\
2 \gamma_{1122} \cos ^{2}(\theta) \sin ^{2}(\theta)+2 \gamma_{1112} \cos (\theta) \sin ^{3}(\theta)+ \\
2 \gamma_{2212} \sin (\theta) \cos ^{3}(\theta)
\end{array}
$$


Furthermore, the Euler-Lagrange equation is rewritten in the form

$$
\begin{array}{r}
c-1-4 l_{0}^{2} \Delta c+4 l_{0}^{4} \sum_{i j k l} \gamma_{i j k l} \frac{\partial^{4} c}{\partial x_{i} \partial x_{j} \partial x_{k} \partial x_{l}}=0 \Rightarrow \\
c-1-4 l_{0}^{2}\left(\frac{\partial^{2} c}{\partial x_{2_{\theta}}^{2}}\right)+4 l_{0}^{4} \gamma_{\theta}\left(\frac{\partial^{4} c}{\partial x_{2_{\theta}}^{4}}\right)=0
\end{array}
$$

Equation (85) can then be numerically solved subject to the following boundary conditions, i.e.,

$$
\begin{aligned}
& c(0)=0 \\
& \frac{\partial c(0)}{\partial x_{2_{\theta}}}=0 \\
& \frac{\partial c( \pm \infty)}{x_{2_{\theta}}}\left(\approx \frac{\partial c\left( \pm x_{l b}\right)}{\partial x_{2_{\theta}}}\right)=0 \\
& \frac{\partial^{2} c( \pm \infty)}{\partial x_{2_{\theta}}^{2}}\left(\approx \frac{\partial^{2} c\left( \pm x_{l b}\right)}{\partial x_{2_{\theta}}^{2}}\right)=0
\end{aligned}
$$

where $x_{l b}$ is the distance from the boundary, assuming that $x_{l b}=20 l_{0}$. Finally, the surface energy density is numerically evaluated as

$$
\mathcal{G}_{c}(\theta)=\int_{-\infty}^{+\infty} \overline{\mathcal{G}}_{c} \mathcal{Z}_{c, A n i s} d x_{2_{\theta}} \approx \int_{-x_{l b}}^{+x_{l b}} \overline{\mathcal{G}}_{c} \mathcal{Z}_{c, A n i s} d x_{2_{\theta}}
$$

The maximum and minimum values of $\mathcal{G}_{c}(\theta)$ for $\theta \in[0,2 \pi]$ define the $\mathcal{G}_{c_{\max }}$ and $\mathcal{G}_{c_{\text {min }}}$ respectively. The polar plot of surface energy density $\mathcal{G}_{c}(\theta)$ can be rotated by angle $\phi$ through relation (88) below

$$
\gamma_{\phi}=Q_{\phi} \gamma \boldsymbol{Q}_{\phi}^{T}
$$

The rotation matrix $\boldsymbol{Q}_{\phi}$ is defined as

$$
\boldsymbol{Q}_{\phi}=\left[\begin{array}{ccc}
c^{2} & s^{2} & -2 c s \\
s^{2} & c^{2} & 2 c s \\
c s & -c s & c^{2}-s^{2}
\end{array}\right]
$$

where $c=\cos (\phi)$ and $s=\sin (\phi)$. The angle $\phi$ goes clockwise. In the cases of cubic and orthotropic symmetry the 4 -th order tensor $\gamma$ is expressed, in global axes, as

$$
\boldsymbol{\gamma}=\left[\begin{array}{lll}
\gamma_{1111} & \gamma_{1122} & \gamma_{1112} \\
\gamma_{2211} & \gamma_{2222} & \gamma_{2212} \\
\gamma_{1211} & \gamma_{1222} & \gamma_{1212}
\end{array}\right]=\left[\begin{array}{ccc}
\gamma_{11} & \gamma_{12} & 2 \gamma_{14} \\
\gamma_{12} & \gamma_{22} & 2 \gamma_{24} \\
2 \gamma_{14} & 2 \gamma_{24} & 4 \gamma_{44}
\end{array}\right]
$$

Acknowledgements The research described in this paper has been financed by the University of Nottingham through the Dean of Engineering Prize, a scheme for pump priming support for early career academic staff. The authors are grateful to the University of Nottingham for access to its high performance computing facility. 


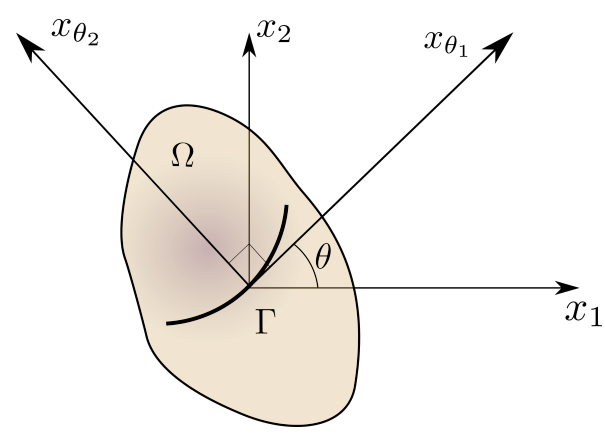

Fig. 20 Coordinate system transformation.

\section{References}

1. Ambati, M., Kruse, R., De Lorenzis, L.: A phase-field model for ductile fracture at finite strains and its experimental verification. Computational Mechanics 57(1), 149167 (2016)

2. Ambrosio, L., Tortorelli, V.M.: Approximation of functional depending on jumps by elliptic functional via t-convergence. Communications on Pure and Applied Mathematics 43(8), 999-1036 (1990)

3. Bandara, S., Soga, K.: Coupling of soil deformation and pore fluid flow using material point method. Computers and Geotechnics 63, 199-214 (2015)

4. Bardenhagen, S., Kober, E.: The generalized interpolation material point method. Computer Modeling in Engineering and Sciences 5(6), 477-495 (2004)

5. Bardenhagen, S.G., Nairn, J.A., Lu, H.: Simulation of dynamic fracture with the Material Point Method using a mixed J-integral and cohesive law approach. International Journal of Fracture 170(1), 49-66 (2011)

6. Bathe, K.J.: Finite element procedures. Prentice Hall, Upper Saddle River, NJ. (2007)

7. Batra, R., Zhang, G.: Search algorithm, and simulation of elastodynamic crack propagation by modified smoothed particle hydrodynamics (MSPH) method. Computational Mechanics 40(3), 531-546 (2007)

8. Bobaru, F., Hu, W.: The Meaning, Selection, and Use of the Peridynamic Horizon and its Relation to Crack Branching in Brittle Materials. International Journal of Fracture 176(2), 215-222 (2012)

9. Borden, M.J., Hughes, T.J., Landis, C.M., Verhoosel, C.V.: A higher-order phase-field model for brittle fracture: Formulation and analysis within the isogeometric analysis framework. Computer Methods in Applied Mechanics and Engineering 273, 100-118 (2014)

10. Borden, M.J., Verhoosel, C.V., Scott, M.A., Hughes, T.J., Landis, C.M.: A phase-field description of dynamic brittle fracture. Computer Methods in Applied Mechanics and Engineering 217-220, 77-95 (2012)

11. Bourdin, B., Francfort, G.A., Marigo, J.J.: The Variational Approach to Fracture. Journal of Elasticity 91(1-3), 5-148 (2008)

12. Clayton, J., Knap, J.: Phase field modeling and simulation of coupled fracture and twinning in single crystals and polycrystals. Computer Methods in Applied Mechanics and Engineering 312, 447-467 (2016)

13. Daphalapurkar, N.P., Lu, H., Coker, D., Komanduri, R.: Simulation of dynamic crack growth using the generalized interpolation material point (GIMP) method. International Journal of Fracture 143(1), 79-102 (2007)

14. Francfort, G.A., Marigo, J.J.: Revisiting brittle fracture as an energy minimization problem. Journal of the Mechanics and Physics of Solids 46(8), 1319-1342 (1998)

15. Griffith, A.A.: The phenomena of rupture and flow in solids. Philosophical Transactions of the Royal Society of London A 221, 163-198 (1921) 
16. Gültekin, O., Dal, H., Holzapfel, G.A.: A phase-field approach to model fracture of arterial walls: theory and finite element analysis. Computer Methods in Applied Mechanics and Engineering 312, 542-566 (2016)

17. Homel, M.A., Herbold, E.B.: Field-gradient partitioning for fracture and frictional contact in the material point method. International Journal for Numerical Methods in Engineering 109(7), 1013-1044 (2017)

18. Hughes, T., Reali, A., Sangalli, G.: Efficient quadrature for NURBS-based isogeometric analysis. Computer Methods in Applied Mechanics and Engineering 199(5-8), 301-313 (2010)

19. Jassim, I., Stolle, D., Vermeer, P.: Two-phase dynamic analysis by material point method. International Journal for Numerical and Analytical Methods in Geomechanics 37(15), 2502-2522 (2013)

20. Kakouris, E.G., Triantafyllou, S.P.: Phase-Field Material Point Method for Brittle Fracture. International Journal for Numerical Methods in Engineering (2017). DOI $10.1002 /$ nme.5580

21. Li, B., Peco, C., Milln, D., Arias, I., Arroyo, M.: Phase-field modeling and simulation of fracture in brittle materials with strongly anisotropic surface energy. International Journal for Numerical Methods in Engineering 102(3-4), 711-727 (2015)

22. Li, T., Marigo, J.J., Guilbaud, D., Potapov, S.: Gradient damage modeling of brittle fracture in an explicit dynamics context. International Journal for Numerical Methods in Engineering 108(11), 1381-1405 (2016)

23. Miehe, C., Aldakheel, F., Raina, A.: Phase field modeling of ductile fracture at finite strains: A variational gradient-extended plasticity-damage theory. International Journal of Plasticity 84, 1-32 (2016)

24. Miehe, C., Hofacker, M., Welschinger, F.: A phase field model for rate-independent crack propagation: Robust algorithmic implementation based on operator splits. Computer Methods in Applied Mechanics and Engineering 199(45-48), 2765-2778 (2010)

25. Miehe, C., Welschinger, F., Hofacker, M.: Thermodynamically consistent phase-field models of fracture: Variational principles and multi-field FE implementations. International Journal for Numerical Methods in Engineering 83(10), 1273-1311 (2010)

26. Nairn, J.A.: Material Point Method Calculations with Explicit Cracks. Computer Modeling in Engineering and Sciences 4(6), 649-664 (2003)

27. Nairn, J.A., Hammerquist, C., Aimene, Y.E.: Numerical Implementation of Anisotropic Damage Mechanics. International Journal for Numerical Methods in Engineering (2017). DOI 10.1002/nme.5585

28. Nguyen, V.P., Rabczuk, T., Bordas, S., Duflot, M.: Meshless methods: A review and computer implementation aspects. Mathematics and Computers in Simulation 79(3), 763-813 (2008)

29. Sadeghirad, A., Brannon, R., Guilkey, J.: Second-order convected particle domain interpolation (CPDI2) with enrichment for weak discontinuities at material interfaces. International Journal for Numerical Methods in Engineering 95(11), 928-952 (2013)

30. Sadeghirad, A., Brannon, R.M., Burghardt, J.: A convected particle domain interpolation technique to extend applicability of the material point method for problems involving massive deformations. International Journal for Numerical Methods in Engineering 86(12), 1435-1456 (2011)

31. Sanchez, J., Schreyer, H., Sulsky, D., Wallstedt, P.: Solving quasi-static equations with the material-point method. International Journal for Numerical Methods in Engineering 103(1), 60-78 (2015)

32. Scholtès, L., Donzé, F.V.: Modelling progressive failure in fractured rock masses using a 3D discrete element method. International Journal of Rock Mechanics and Mining Sciences 52, 18-30 (2012)

33. Schreyer, H., Sulsky, D., Zhou, S.J.: Modeling delamination as a strong discontinuity with the material point method. Computer Methods in Applied Mechanics and Engineering 191(23-24), 2483-2507 (2002)

34. Shanthraj, P., Svendsen, B., Sharma, L., Roters, F., Raabe, D.: Elasto-viscoplastic phase field modelling of anisotropic cleavage fracture. Journal of the Mechanics and Physics of Solids 99, 19-34 (2017) 
35. Steffen, M., Kirby, R.M., Berzins, M.: Analysis and reduction of quadrature errors in the material point method (MPM). International Journal for Numerical Methods in Engineering 76(6), 922-948 (2008)

36. Steffen, M., Wallstedt, P.M., Guilkey, J., Kirby, R., Berzins, M.: Examination and analysis of implementation choices within the material point method (MPM). Computer Modeling in Engineering and Sciences 31(2), 107-127 (2008)

37. Sulsky, D., Chen, Z., Schreyer, H.L.: A particle method for history-dependent materials. Computer Methods in Applied Mechanics and Engineering 118(1-2), 179-196 (1994)

38. Sulsky, D., Kaul, A.: Implicit dynamics in the material-point method. Computer Methods in Applied Mechanics and Engineering 193(1214), 1137-1170 (2004)

39. Sulsky, D., Schreyer, L.: MPM simulation of dynamic material failure with a decohesion constitutive model. European Journal of Mechanics-A/Solids 23(3), 423-445 (2004)

40. Ting, T.C.t.: Anisotropic Elasticity: Theory and Applications. Oxford University Press, New York (1996)

41. Yang, P., Gan, Y., Zhang, X., Chen, Z., Qi, W., Liu, P.: Improved decohesion modeling with the material point method for simulating crack evolution. International Journal of Fracture 186(1), 177-184 (2014) 